University of Tennessee Health Science Center

UTHSC Digital Commons

\title{
Quantifying Joint Coordination Variability in Anterior Cruciate Ligament-Reconstructed Individuals During Walking
}

Kylie Davis

University of Tennessee Health Science Center

Follow this and additional works at: https://dc.uthsc.edu/dissertations

Part of the Diagnosis Commons, Investigative Techniques Commons, Other Analytical, Diagnostic and Therapeutic Techniques and Equipment Commons, and the Therapeutics Commons

\section{Recommended Citation}

Davis, Kylie (http://orcid.org/0000-0002-5118-5979), "Quantifying Joint Coordination Variability in Anterior Cruciate Ligament-Reconstructed Individuals During Walking" (2017). Theses and Dissertations (ETD). Paper 441. http://dx.doi.org/10.21007/etd.cghs.2017.0433.

This Thesis is brought to you for free and open access by the College of Graduate Health Sciences at UTHSC Digital Commons. It has been accepted for inclusion in Theses and Dissertations (ETD) by an authorized administrator of UTHSC Digital Commons. For more information, please contact jwelch30@uthsc.edu. 


\title{
Quantifying Joint Coordination Variability in Anterior Cruciate Ligament- Reconstructed Individuals During Walking
}

\begin{abstract}
The knee is the second most common joint to sustain injury. An estimated 200,000 anterior cruciate ligament (ACL) ruptures occur each year in the United States alone, and about 100,000 ACL reconstruction (ACLR) surgeries are performed annually. There is a significant risk of developing osteoarthritis of the knee after incurring an ACL injury, and the incidence of ipsilateral or contralateral injury is six times greater in individuals who have a surgically repaired ACL. Past studies have analyzed kinetic and kinematic characteristics of individual lower extremity joints to reveal differences between subjects with and without ACLR. Despite reports of altered kinematic performance in individuals with ACLR compared to healthy controls, most of the analyses did not evaluate coordinative function, and thus neglected to consider how the lower limb acts as a linked chain. Therefore, the present study used a method based on dynamical systems theory to quantify coordination and account for the interaction between joints in the lower extremity. The purpose of the study was to quantify and compare joint coordination variability and joint coordination patterns between individuals with ACLR and matched controls. Institutional Review Board (IRB) approval was obtained prior to data collection, and all subjects signed an informed consent form. Twenty subjects (nine females, eleven males; body mass index (BMI) $25 \pm 3.5 \mathrm{~kg} / \mathrm{m} 2$ ) who had undergone unilateral ACLR (thirteen right, seven left) and been cleared to return to full activity were compared to twenty control subjects matched by gender and BMI (nine females, eleven males; BMI $22.4 \pm 2.4 \mathrm{~km} / \mathrm{m} 2$ ). Kinematic and kinetic data during walking were collected in the UTHSC Motion Analysis Laboratory. A vector coding technique was used to calculate coupling angles for six joint couplings involving the hip, knee, and ankle across four periods within the stance phase. Joint coordination variability was defined as the standard deviation of the coupling angle between trials within a subject, and joint coordination patterns were based on coupling angle magnitude. Individuals with ACLR exhibited increased joint coordination variability and altered joint coordination patterns compared to the matched controls during the stance phase of walking. These results suggested that coordinative function may not be fully restored in individuals with ACLR following rehabilitation. Increased coordination variability from a normal, or optimal amount as well as altered coordination patterns may result from a deficit in sensorimotor control, and represent risk of re-injury. Further investigation that is prospective, focuses primarily on hip-knee coupled motion in frontal and transverse planes, and includes assessment of EMG in addition to kinematics may contribute relevant information for improving ACL injury prevention and rehabilitation.
\end{abstract}

\section{Document Type}

Thesis

\section{Degree Name}

Master of Science (MS)

\section{Program}

Biomedical Engineering

Research Advisor

Brooke A. Sanford, Ph.D.

Keywords

Coordination, Variability, Vector coding 


\section{Subject Categories}

Analytical, Diagnostic and Therapeutic Techniques and Equipment | Diagnosis | Investigative Techniques | Medicine and Health Sciences | Other Analytical, Diagnostic and Therapeutic Techniques and Equipment | Therapeutics 


\title{
Quantifying Joint Coordination Variability in Anterior Cruciate Ligament- Reconstructed Individuals During Walking
}

\author{
A Thesis \\ Presented for \\ The Graduate Studies Council \\ The University of Tennessee \\ Health Science Center
}

\author{
In Partial Fulfillment \\ Of the Requirements for the Degree \\ Master of Science \\ In the Joint Graduate Program in Biomedical Engineering and Imaging \\ From The University of Tennessee \\ And \\ The University of Memphis
}

By

Kylie Davis

May 2017 
Copyright (C) 2017 by Kylie Davis. All rights reserved. 


\section{ACKNOWLEDGEMENTS}

First and foremost, I would like to acknowledge both Dr. Sanford and Dr. Williams for their exceptional guidance along the way to finishing this thesis. I am incredibly fortunate to have been privy to Dr. Williams' mentorship not only during my time in graduate school but also in my undergraduate program. He deserves to be recognized for the knowledge, leadership, and support that he provided to me throughout my university career that went above and beyond what I could have asked for. I must also credit him for my success in earning a Master's Degree; he is responsible for introducing me to what is now my most-liked field of study, as well as opening the door to working with Dr. Sanford. I can't thank Dr. Sanford enough for giving me the opportunity to work as her graduate research assistant. She also deserves recognition for being an approachable adviser that is always willing to listen to and answer questions. I gained a lot from her encouragement, direction, and advice, and she made my experience as a graduate student enjoyable.

Second, I would like to acknowledge Dr. Zucker-Levin for serving on my committee. I must thank her for dedicating precious time and effort to give invaluable feedback that made my thesis better. I would also like to thank the rest of the faculty in the Joint Program in Biomedical Engineering at UTHSC/UM. Lastly, I would like to express my appreciation for the priceless support given to me by Julie Lowell and Meredith Perkins.

Lastly, I would like to thank my family for their love, support, and constant cheering over the last six years. I can't put into words how lucky I am to have been able to pursue my goals with the resources I was given because of my parents' hard work and generosity. I also can't forget the special friendship and encouragement I get from my siblings that have made me a very grateful and happy little sister. I attribute many of my successes to my family, and will continue to strive to make them proud. 


\begin{abstract}
The knee is the second most common joint to sustain injury. An estimated 200,000 anterior cruciate ligament (ACL) ruptures occur each year in the United States alone, and about 100,000 ACL reconstruction (ACLR) surgeries are performed annually. There is a significant risk of developing osteoarthritis of the knee after incurring an ACL injury, and the incidence of ipsilateral or contralateral injury is six times greater in individuals who have a surgically repaired ACL.

Past studies have analyzed kinetic and kinematic characteristics of individual lower extremity joints to reveal differences between subjects with and without ACLR. Despite reports of altered kinematic performance in individuals with ACLR compared to healthy controls, most of the analyses did not evaluate coordinative function, and thus neglected to consider how the lower limb acts as a linked chain. Therefore, the present study used a method based on dynamical systems theory to quantify coordination and account for the interaction between joints in the lower extremity. The purpose of the study was to quantify and compare joint coordination variability and joint coordination patterns between individuals with ACLR and matched controls.
\end{abstract}

Institutional Review Board (IRB) approval was obtained prior to data collection, and all subjects signed an informed consent form. Twenty subjects (nine females, eleven males; body mass index (BMI) $25 \pm 3.5 \mathrm{~kg} / \mathrm{m}^{2}$ ) who had undergone unilateral ACLR (thirteen right, seven left) and been cleared to return to full activity were compared to twenty control subjects matched by gender and BMI (nine females, eleven males; BMI $22.4 \pm 2.4 \mathrm{~km} / \mathrm{m}^{2}$ ). Kinematic and kinetic data during walking were collected in the UTHSC Motion Analysis Laboratory. A vector coding technique was used to calculate coupling angles for six joint couplings involving the hip, knee, and ankle across four periods within the stance phase. Joint coordination variability was defined as the standard deviation of the coupling angle between trials within a subject, and joint coordination patterns were based on coupling angle magnitude.

Individuals with ACLR exhibited increased joint coordination variability and altered joint coordination patterns compared to the matched controls during the stance phase of walking. These results suggested that coordinative function may not be fully restored in individuals with ACLR following rehabilitation. Increased coordination variability from a normal, or optimal amount as well as altered coordination patterns may result from a deficit in sensorimotor control, and represent risk of re-injury. Further investigation that is prospective, focuses primarily on hip-knee coupled motion in frontal and transverse planes, and includes assessment of EMG in addition to kinematics may contribute relevant information for improving ACL injury prevention and rehabilitation. 


\section{TABLE OF CONTENTS}

CHAPTER 1. INTRODUCTION ..................................................................................

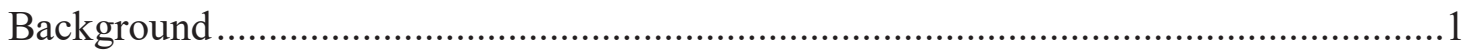

Dynamical Systems Theory ...............................................................................

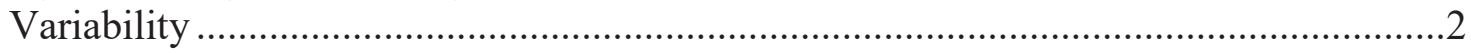

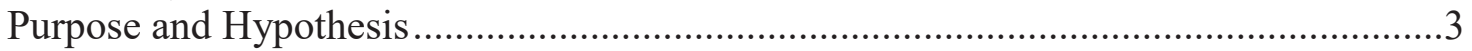

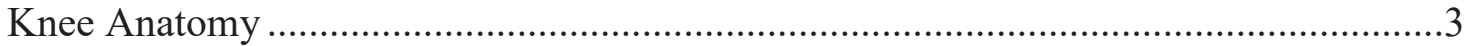

Risk Factors for ACL Injury ........................................................................... 4

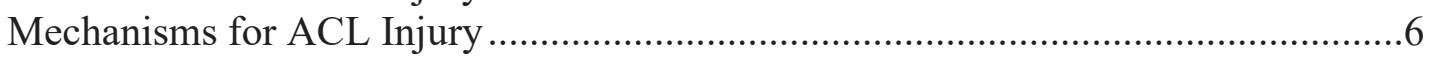

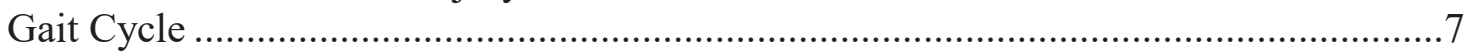

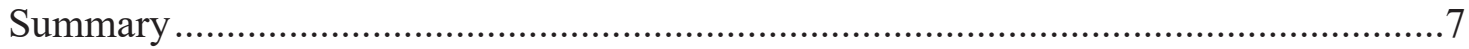

CHAPTER 2. LITERATURE REVIEW ................................................................9

Dynamical Systems Approach to Analyzing Human Movement Coordination ..............9

Relative Phase .................................................................................................... 10

Vector Coding ................................................................................................. 12

Movement Coordination and Coordinative Variability in ACLR Individuals ..............15

CHAPTER 3. METHODOLOGY ..................................................................17

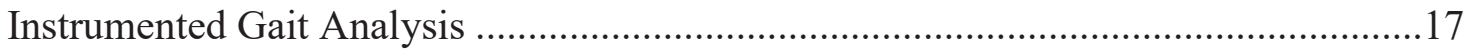

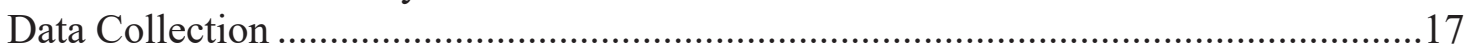

3D Modeling and Joint Angle Calculations ............................................................... 19

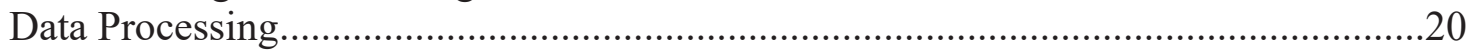

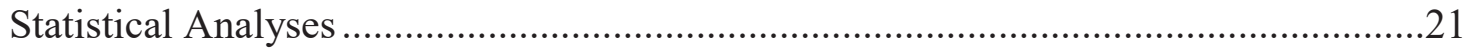

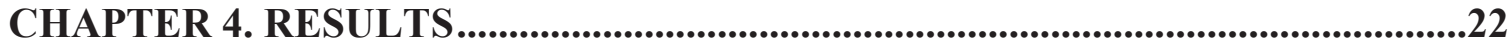

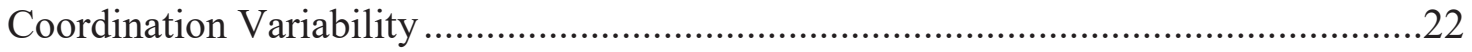

Coordination Phase ...........................................................................................22

CHAPTER 5. DISCUSSION .......................................................................................28

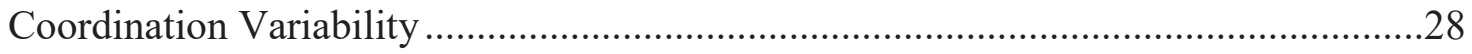

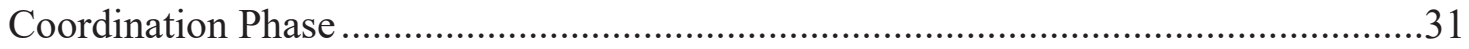

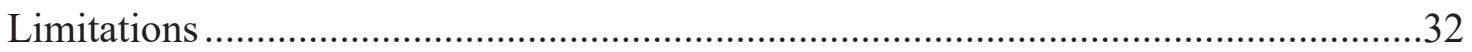

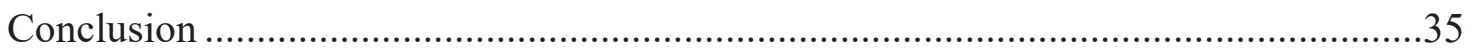

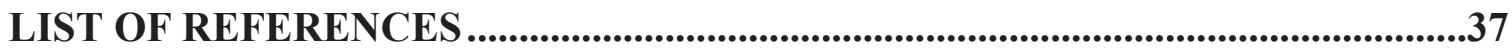

APPENDIX. SUMMARY OF JOINT COORDINATION AND JOINT

COORDINATION VARIABILITY MEASURES.....................................................44

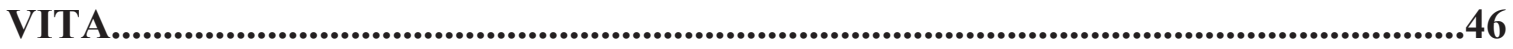




\section{LIST OF TABLES}

Table 2-1. Categories used to describe coordination patterns ....................................14

Table 3-1. Mean \pm SD for subject metrics for the ACLR and Control groups ................18

Table 4-1. Joint coordination variability in the reconstructed and matched limb for ACLR versus Control .........................................................................23

Table 4-2. Joint coordination variability in the contralateral limb for ACLR versus the reconstructed-matched limb for Control

Table 4-3. Joint coordination phase magnitude $\left(^{\circ}\right)$ and classification for the reconstructed and matched limbs in ACLR versus Control........................26

Table A-1. Summary of joint coordination variability measures for all joint couplings and stance periods

Table A-2. Summary of joint coordination patterns for all joint couplings and stance periods 


\section{LIST OF FIGURES}

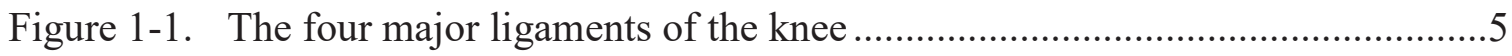

Figure 1-2. The gait cycle divided into sub-phases ..................................................

Figure 4-1. Angle-angle plot of the hip rotation/knee abduction-adduction coupling for one matched pair across the stance phase ...........................................24

Figure 4-2. Coupling angle for hip rotation/knee abduction-adduction across the stance phase.....

Figure 4-3. Angle-angle plot of the hip rotation/knee rotation coupling for one matched pair across the stance phase .......................................................2.

Figure 4-4. Coupling angle for hip rotation/knee rotation across the stance phase.........25

Figure 4-5. Ensemble average showing the coupling angle variability in hip rotation/knee abduction-adduction across the stance phase .26 


\section{CHAPTER 1. INTRODUCTION}

\section{Background}

The knee is the second most common joint to sustain injury [1]. It is the most prevalently injured joint in adolescent athletes [2], and the foremost cause for sportrelated surgeries[1]. An estimated 200,000 anterior cruciate ligament (ACL) ruptures occur each year in the United States alone, and about 100,000 ACL reconstruction surgeries are performed annually [3]. The average time to return to sport following ACL reconstruction is between six and twelve months, but Ardern et al. [4] claimed only $82 \%$ of patients who underwent reconstruction were capable of resuming their previous activities, and little more than half regained their pre-injury level of performance. There is also a significant risk of developing osteoarthritis of the knee after incurring an ACL injury [5], and the incidence of ipsilateral or contralateral injury is six times greater in individuals who have a surgically repaired ACL [6]. Further, there is a reported $\$ 2$ billion annual health care cost associated with ACL injury [7]. The physical, emotional, and financial costs related to ACL injury incite researchers to better understand its etiology with the hopes of improving prevention and treatment protocols.

\section{Dynamical Systems Theory}

Past studies have analyzed kinetic and kinematic characteristics of individual lower extremity joints to reveal differences between ACL-reconstructed (ACLR) and uninjured individuals, and consistent findings include decreased knee flexion [8], increased knee abduction [8-11], increased hip adduction [12, 13], flexion [9] and internal rotation [10], and increased ankle eversion [14] during tasks like walking, running, and landing. These results are evidence that differences in joint function could exist between ACLR and uninjured populations. Most traditional analyses have not evaluated coordinative function, and therefore, have neglected to consider how the lower limb acts as a linked chain. A recent alternative method for studying ACL injury is based on dynamical systems theory, which examines the interaction between two joints or segments.

The dynamical systems theory proposes that a healthy motor system has redundant degrees of freedom (DOF). These DOF provide multiple pathways to perform a task and are controlled by coordinative structures. Accordingly, Bernstein [15] defined coordination as the process of mastering redundant DOF to produce a controllable system. The dynamical systems approach to studying human movement was established from this concept and attempts to quantify coordination. The same DOF may be used to execute different movement patterns, and different DOF may be used to achieve the same patterns [16]. Variability in coordination is a result of the infinite number of combinations employed by available DOF to complete a task, and is possibly an essential component of movement that allows for stability and flexibility. Researchers have hypothesized that there is an optimal amount of variability present in any motor system 
that differentiates between the ability to adjust to environmental instability and the risk for injury, but have yet to discover a conclusive range. Todorov and Jordan [17] describe the complexity of coordination by stating behavioral goals are attained repeatedly and reliably with movements that are seldom reproducible in their detail.

\section{Variability}

Two definitions of variability are commonly used in biomechanics; end-point variability and coordination variability. End-point variability is goal-oriented and measures an outcome, whereas coordinative variability relates to the process by which an outcome was produced over a number of repetitions [18]. Bartlett [19] studied the effect of varying release parameters on the flight of a javelin, and found that infinite combinations of these parameters produced the same range of throwing distance. The same author reported mixed arm movement patterns among twelve men's javelin finalists at a championship meet, as well as intra-thrower kinematic differences at the shoulder and elbow in elite javelin throwers. It was concluded that outcome consistency is not directly correlated with movement consistency, and variant movement patterns are inevitable even in high-level performance. Moreover, increased coordinative variability has been observed in expert performers in sport [20]. This study quantified lower extremity intra-limb coordination variability in triple jumpers to determine whether skill had an impact on variability. All jumpers were considered experts, but were divided into least, intermediate, and most skilled categories based on global ranking. The authors hypothesized that skill would have a direct relationship on coordination variability such that as skill increased, variability would also increase. Results showed that intermediate jumpers demonstrated the lowest coordination variability, while the least and most skilled jumpers had higher variability. Therefore, the pattern of change in coordination variability was U-shaped relative to skill level. Although the least skilled jumpers displayed similar high variability like the expert jumpers, this was explained using a traditional motor learning perspective, which states that higher variability is observed in the early stages of skill development. As such, despite being an expert, the least skilled jumpers could be in the early stage of learning how to coordinate the complex movement of a triple jump at a higher level of competition. Lastly, reduced coordinative variability has been associated with orthopedic disorders [21]. Hamill et al. [22] measured variability in lower extremity couplings to discriminate between symptomatic individuals with patellofemoral pain (PFP) and asymptomatic individuals during treadmill running. Both pattern and magnitude differences were observed between groups where symptomatic individuals displayed less variability and the largest differences occurred during the transitions between the stance and swing phases. Therefore, a decrease in variability was indicative of a pathological system. Concurrently, another researcher [23] investigated the variability of joint coordination in individuals with PFP during treadmill running and reported less variability in the injured group compared to healthy participants. 


\section{Purpose and Hypothesis}

The purpose of this thesis was to quantify and compare joint coordination variability in ACLR individuals and uninjured matched controls during walking. A vector coding method was used to analyze lower extremity kinematics and obtain a measure of joint coordination variability, expressed as the standard deviation about the mean. Within-subject variability was hypothesized to be lower in the ACLR group based on a review of the literature that revealed decreased variability in pathological systems [2426].

A secondary aim of this work was to compare joint coordination phases between the ACLR and uninjured groups to quantify any differences in relative joint motion patterns. Consistent with the idea that the healthy components of a movement system will adapt their function to compensate for dysfunction at an injured site, the ACLR group was expected to demonstrate altered coordination phase patterns compared to the control group. Gribbin et al. [27] examined differences in hip-knee joint coupling during gait after ACLR and reported larger coupling angles in ACLR individuals compared to healthy subjects. This was interpreted as the hip having decreased contribution to joint movement relative to the knee. The authors suspected that the hip acted as a stabilizer in ACLR individuals as a response to increased knee motion. Therefore, coupling angles were hypothesized to be increased in ACLR individuals compared to those in healthy individuals.

This thesis is divided into five chapters. Chapter 1 consists of background information pertaining to ACL injury, including its incidence and physical, emotional and economic impacts. Pertinent anatomy, risk factors, and mechanisms related to ACL injury, as well as a description of the motion capture system used to collect data are also covered in Chapter 1. Chapter 2 entails an extensive review of the literature and provides rationale for this research study. Detailed methods are outlined in Chapter 3 and the results are reported in Chapter 4, including statistical analyses. Chapter 5 is a discussion about the interpretation of the results, and offers a conclusion to the stated hypothesis based on these findings.

\section{Knee Anatomy}

The human knee comprises two joints: the femorotibial joint and the patellofemoral joint. It is often simplified as a hinge joint, but in reality the knee is a more intricate construct that experiences translation and undergoes rotation in multiple planes. Knee function is complex involving the interaction of several bones, muscles, and ligaments to provide joint motion and stability. The bones of the knee joint consist of the femur (distal portion), tibia (proximal portion), and patella, and the main muscles acting to flex and extend it are the quadriceps femoris, biceps femoris, and gastrocnemius. The four major ligaments are the medial and lateral collateral ligaments and the anterior and posterior cruciate ligaments. The medial collateral ligament controls lateral motion of the knee (valgus) while the lateral collateral ligament controls medial motion of the knee 
(varus). The cruciate ligaments crisscross each other such that the anterior cruciate ligament (ACL) crosses in front of the posterior cruciate ligament (PCL), and are primarily responsible for controlling anterior-posterior movement of the knee. Figure 1-1 is a schematic showing the organization of the four major ligaments in the knee.

An ACL tear is among the most common knee injuries and results in loss of joint stability. Specifically, the ACL attaches to the posteromedial surface of the lateral femoral condyle and crosses the knee joint diagonally to insert anterior and lateral to the tibial spine. It consists of two bundles (anteromedial and posterolateral) that provide $85 \%$ of the restraining force against anterior translation of the tibia in relation to the femur [28]. The ACL also prevents excessive varus-valgus stresses (abduction-adduction), and contributes to the control of hyperflexion and hyperextension of the knee. An ACL tear is among the most common knee injuries and results in loss of joint stability.

In the femorotibial joint, the medial and lateral femoral condyles articulate with the medial and lateral tibial plateaus, respectively. The intercondylar notch is a deep notch between the medial and lateral femoral epicondyles that joins the knee. Medial and lateral menisci, crescent-shaped cartilage between the femur and tibia, act as shock absorbers, and provide lubrication, force transmission, stability, and also facilitate rotation of the knee. The patellofemoral joint involves the patella and femoral trochlea, and its main function is knee stability. The patella acts as an anatomical pulley by increasing the distance (moment arm) of applied quadriceps force from the axis of knee rotation, thus reducing the quadriceps force required to extend the knee.

\section{Risk Factors for ACL Injury}

The risk factors related to ACL injury are commonly divided into anatomical, environmental, and biomechanical or neuromuscular categories. Based on a review by Smith et al. [29, 30], anatomical variables that increase the risk of ACL injury include decreased intercondylar notch size, increased slope of the tibial plateaus, and increased knee laxity. Increased navicular drop and foot pronation have also been identified as possible contributors to injury [14, 31]. Ground surface type, weather, and footwear have been considered as environmental factors, but like anatomical factors, are secondary to the major emphasis placed on biomechanical aspects affecting the risk for ACL injury. Many biomechanical risk factors such as decreased knee flexion [8], increased knee abduction [9, 11], increased hip adduction [12], flexion [9] and internal rotation [10], increased tibial rotation [14], and increased ankle eversion [14] may increase the risk of ACL injury. A position in which the knee is considered at risk for ACL injury has been identified consistently [32-34] where the knee is near full extension, the tibia is externally rotated, and the foot is landed during a deceleration. Additionally, foot landing position, specifically toe-in, has recently been reported as contributing to the aforementioned biomechanical risk factors [35]. In summary, factors that cause increased valgus stress $[11,36]$, tibial translation, and/or excessive loading on the ACL may increase ACL injury risk. 


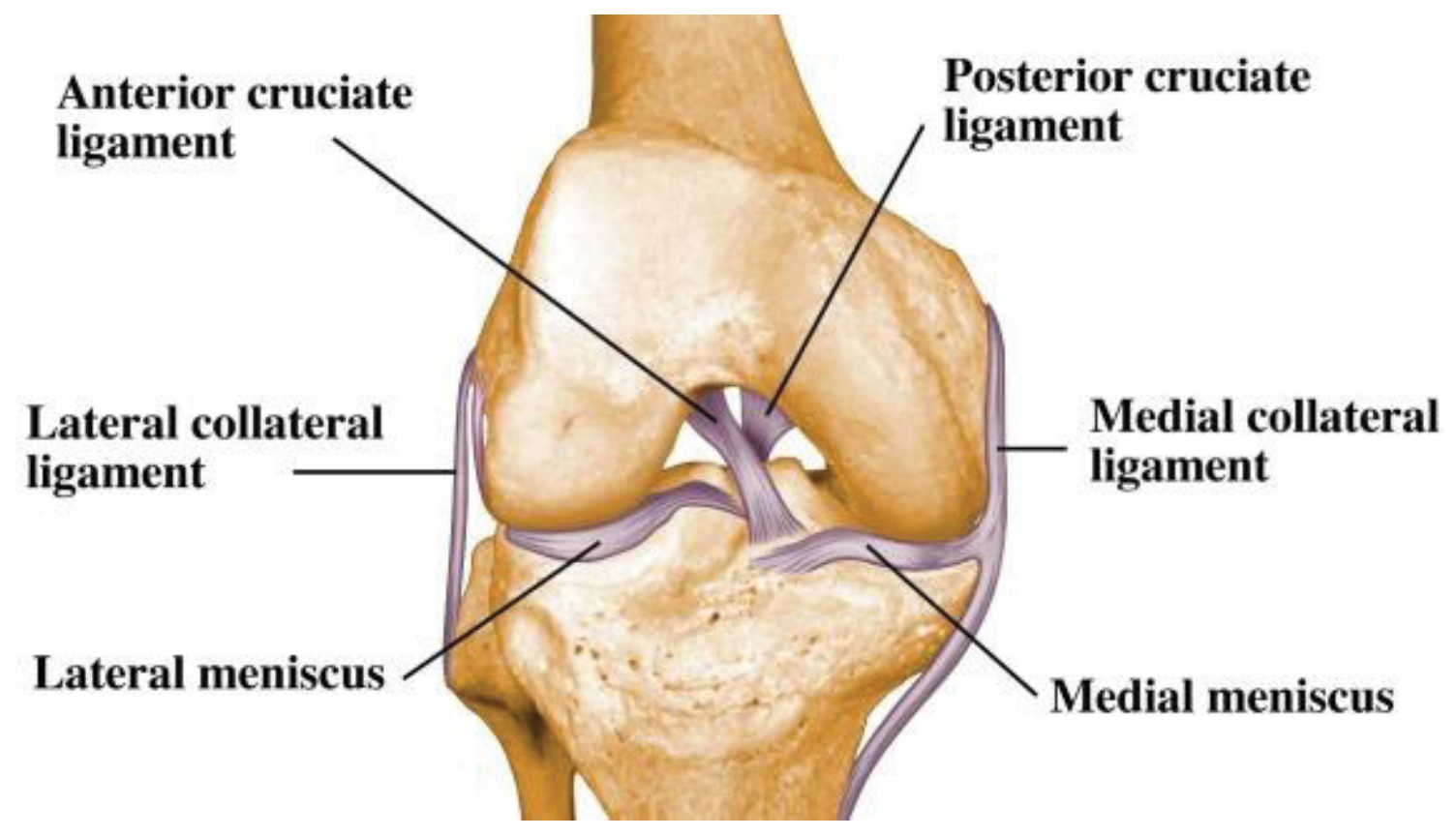

Figure 1-1. The four major ligaments of the knee Reprinted with permission from New Health Advisor: Ligaments of the knee. Accessed March 16, 2017, from http://www.newhealthadvisor.com/ligaments-of-the-knee.html 


\section{Mechanisms for ACL Injury}

Most ACL injuries occur through noncontact mechanisms [34]. Common movement patterns that involve injury to the ACL are landing, pivoting, and cutting maneuvers. Olsen et al. [37] observed two main mechanisms in a video analysis of handball athletes. The first, which occurred more frequently, was the combination of a landing and subsequent cutting move characterized by a large valgus knee moment and rotation of the tibia near full knee extension. The knee moment is a product of the ground reaction force (GRF) and distance from the GRF vector to the center of rotation of the knee. The second mechanism was a single leg landing that also exhibited increased knee valgus as well as external tibial rotation near full knee extension. Ireland [38] supported these findings by suggesting hip adduction and internal rotation, knee valgus, and tibial external rotation as contributors to ACL injury. Yu and Garrett [39] included increased posterior ground reaction force (GRF) and quadriceps contraction as other relevant ACL loading mechanisms pertaining to injury.

It is generally accepted than an injury to the ACL is unlikely to result from a single dysfunction isolated at one joint [40]. The lower extremity kinetic chain comprises the hip, knee, and ankle which work together to produce movement. In the case of impaired or abnormal function at one joint, one or both of the other joints in the chain may adapt its motion as a compensation mechanism to maintain regular overall movement. Compensatory mechanisms at the hip or ankle may, therefore, reveal pathology at the knee. Conversely, abnormal motion at the hip or ankle may contribute to knee pathology. Several differences in hip and ankle function have already been identified relating to injury at the knee. Decreased hip abduction and external rotation, which are signs of hip weakness, influence the development of patellofemoral pain as reported by several researchers [41-44]. Noehren [45] associated increased peak hip adduction angle to the development of iliotibial band syndrome. Consistent with these risk factors related to ACL injury, many researchers have offered evidence of greater hip adduction and internal rotation during a variety of dynamic tasks in females, who are at a higher risk for ACL injury compared to males [46-48]. Further, Lawrence [49] divided female participants according to hip adductor and hip external rotator strength, and those considered as stronger demonstrated lower ground reaction forces during landing in addition to a lower external knee adduction moment. Similarly, Devita and Skelly [50] examined how forces are dissipated throughout the kinetic chain during landing and found that in a stiff landing, indicative of decreased ankle dorsiflexion, increased extensor moment at the hip and flexor moment at the knee were observed. Reduced ankle dorsiflexion has also been linked to patellar tendinopathy [51,52], and other studies have highlighted limited ankle range of motion (ROM) in individuals with excessive medial knee displacement, or knee valgus $[53,54]$. These previously documented abnormal or compensatory joint functions support the need for analyzing ankle and hip kinematics related to knee pathology. 


\section{Gait Cycle}

The gait cycle can be divided into several phases and sub-phases, as depicted in Figure 1-2. In normal gait, the knee is near full extension $\left(0-5^{\circ}\right.$ of flexion $)$ at initial contact and flexes to about $15^{\circ}$ during the loading response phase. As the gait cycle progresses through mid-stance and terminal stance, the knee moves toward extension then returns to flexion before toe off. The hip is flexed to about $30^{\circ}$ at initial contact and proceeds to extend until it reaches maximum extension at terminal stance and begins flexion when the heels rise just prior to toe off. Hip adduction occurs during the early portion of stance, and hip internal rotation is at a maximum around mid-stance. The ankle is in a neutral position at initial contact but plantarflexes during the loading response phase. The ankle transitions to dorsiflexion in terminal stance and returns to plantarflexion at toe off.

Noehren studied gait in females with ACLR and found decreased hip flexion during walking [55]. Czamara [56] reported excessive hip internal rotation across the gait cycle and increased external rotation of the ACLR knee compared to the control knee. Another study [57] compared hip and knee kinematics and kinetics in ACLR individuals who passed and failed return to sport (RTS) criteria, and found several differences. Those who failed had larger knee flexion in the uninvolved limb at initial contact during walking. No significant differences in hip flexion angle were observed, however, kinetic analysis showed that individuals in the fail group generated power with their involved hip but absorbed power in their uninvolved hip during early stance.

\section{Summary}

It is well documented that ACL injury is common and has an unfavorable outcome; many cases involve development of osteoarthritis, re-injury, or failure to return to sport or the pre-injury state. While a number of studies have successfully identified differences in kinetic or kinematic performance between ACLR and healthy populations, these findings have been limited to single joint function and neglected to consider the lower extremity as a linked system. The dynamical systems approach to kinematic analysis evaluates the interaction among joints and thus may provide a more complete assessment of function. Specifically, variability in joint coordination may reveal system constraints or limited access to or use of different movement strategies. Examination of coordination phases may complement variability measures by revealing compensatory mechanisms and describing joint excursion. It is known that individuals with ACLR are susceptible to developing arthritis or incurring a second injury, but this risk has yet to be fully understood. Therefore, the goal of this thesis was to quantify joint coordination in individuals with ACLR and contribute findings that may add to our current understanding of biomechanical function related to ACL injury. 


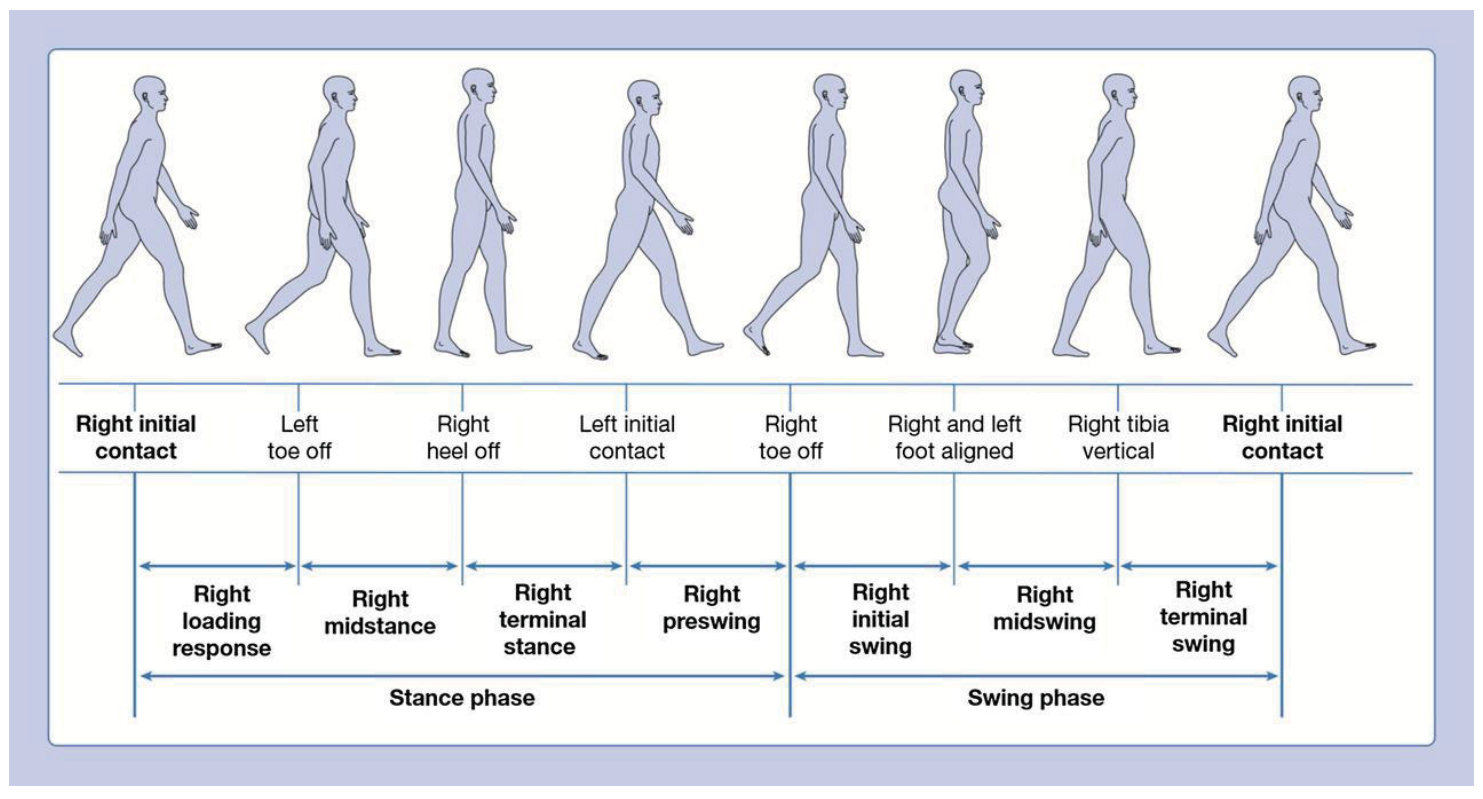

Figure 1-2. The gait cycle divided into sub-phases

Reprinted with permission:

Esquenazi, A. Gait analysis: technology and clinical application. In: Braddom RL, editor. Physical Medicine and Rehabilitation. 3rd ed. Philadelphia: Saunders/Elsevier; 2007. p.93-110. 


\section{CHAPTER 2. LITERATURE REVIEW}

\section{Dynamical Systems Approach to Analyzing Human Movement Coordination}

The dynamical systems theory describes a movement system as having many subsystems, or components, that interact through self-organization to produce a movement pattern for a given task. The complexity of such a system lies within its control; inertia, reaction forces, initial postural conditions, and muscle forces act to produce movements in a body that comprises several mechanical degrees of freedom (DOF) [58]. Bernstein [15] stated that the process of mastering these redundant DOF is the fundamental concept behind coordination and producing a controllable system. Coordinative structures are groupings of muscles spanning several joints into single units that act independently and constrain the DOF within a system, thereby reducing the number of independent parts to be controlled $[58,59]$. An important aspect of coordinative structures that relates to the work presented in this thesis is the relationship between sub-structures such that if one is dysfunctional or introduces an error to the system, the other(s) adjusts their function to minimize this error and preserve the initial movement goal [59]. This compensatory mechanism allows the system to have flexibility and stability in response to perturbation. Another characteristic of coordinative structures is the ability to arrange different DOF in the same manner to accomplish the same task, or organize the same DOF in different configurations to accomplish different tasks $[16,58]$. Variation in resolving the coordination of a system is potentially a vital element of movement, and scientists have proposed an optimal amount of movement variability relating to skill development and a healthy state [60].

Movement variability has previously been associated with error and related to the amount of noise present in a motor system [61]. However, the emergence of the dynamical systems theory led scientists to discover that movement variability is actually an inherent part of the signal and cannot be removed, unlike measurement noise that can be attenuated [62]. Therefore, variability represents an integral component of human motor behavior [61]. It is important to note that there are two distinct measures of variability that result in opposite interpretations of the state or performance of a movement system. End-point variability is a measure of outcome and is used as an indicator of skill level. Low end-point variability is representative of a skilled or expert performance, and this characterization was previously used to describe skilled motor performance as also having low variability or consistent patterns of movement. A frequently referenced study by Arutyunyan et al. [63] provided evidence to reject that consistency in movement patterns is a defining aspect of expert performance. Novice and expert marksmen pistol shooting were compared, and results showed that the expert had lower variability in the spatial orientation of the pistol barrel but greater variability in shoulder and wrist motion. A different study that evaluated javelin throwing supported the previous author's conclusion that increased variation in movement patterns is related to expertise [19]. Infinite combinations of different release parameters were found to produce the same range of throwing distance, and mixed arm movement patterns were observed between the finalists at a javelin championship meet. Further, intra-thrower 
kinematic differences at the shoulder and elbow were found in elite javelin throwers. These findings show that invariant movement patterns are not achieved even in expert performers, and suggest that coordinative variability is desirable.

An early attempt to measure coordination was made by Grieve [64], who analyzed walking patterns using relative motion plots. A relative motion plot is a graphical representation of the angular displacement over time of two joints, and is often termed angle-angle diagram. While visual inspection of relative motion plots is useful for observing consistency in cycles and similarities in movement patterns, it is largely a qualitative analysis. Hershler and Milner [65] built upon the use of relative motion plots to assess gait, and extracted values for area $(\mathrm{A})$, perimeter $(\mathrm{P})$, and shape $\left(\mathrm{P}_{\mathrm{A}}\right)$ of the closed loop (defined by the ratio of $\mathrm{P}$ to the square root $\mathrm{A}$ ). However, specific geometric shape cannot be inferred from the value of $\mathrm{P}_{\mathrm{A}}$. In an effort to better quantify differences between relative motion plots, Whiting and Zernicke [66] used a chain-encoding procedure that was developed by Freeman [67]. This technique superimposes a grid on the relative motion plot and generates a chain of digital points that approximates the shape of the original analog curve according to an eight-point scale [68]. Integer chains are compared by means of cross-correlation, which produces a recognition coefficient $(\mathrm{R})$ that corresponds to the peak value of the cross-correlation function. Two movement patterns are considered identical when $\mathrm{R}$ is equal to 1 and dissimilar when $\mathrm{R}$ approaches 1. A major limitation of this technique is the requirement for data points to be equally spaced, which is often not seen in gait or most human movement [68]. Also, chainencoding transforms data from a ratio scale to the nominal scale, which may result in loss of information and limits statistical analyses [69]. Sparrow et al. [67] developed a modified encoding technique for which the angle between two consecutive data points is calculated relative to the right horizontal. An adapted version of Freeman's original cross-correlation function was also presented to account for the length between data points and include angular data. Tepavac and Field-Fote [69] employed this vector coding technique as a method that preserves ratio scale data to quantify variability in relative joint motion across multiple cycles of gait.

Another way to measure coordination was established by Kelso [70] based on relative phase. In accordance with the dynamical systems theory and Bernstein's view, Kelso's approach to studying human behavior was based on the concept that patterned behavior is governed by self-organization [16]. In one experiment, index fingers spontaneously transitioned from anti-phase oscillation to in-phase oscillation in response to an increase in frequency. This shift demonstrated traits of nonlinear transitions, and led Kelso to use continuous relative phase analysis to detect stability and transitions in dynamic coordination [70].

\section{Relative Phase}

Another way to quantify coordination is by using relative phase methods. Discrete relative phase (DRP) measures coordination on the basis of a temporal phase relationship between two joints. Unlike in the vector coding technique, the angular excursion of each 
joint of interest is plotted relative to time in separate graphs. The time to a specific event during a movement cycle, such as peak flexion, is obtained for both joints and used along with the cycle period $(\mathrm{T})$ to calculate the DRP angle $\left(\mathrm{DRP}_{\theta}\right)$, which is defined by

\section{Equation 2-1.}

$$
D R P_{\theta}=\frac{t_{1}-t_{2}}{T} \times 360^{\circ}
$$

The output variable, $\mathrm{DRP}_{\theta}$, provides the relative timing for two joints in reaching a specified discrete event. A DRP ${ }_{\theta}$ equal to $0^{\circ}$ or $360^{\circ}$ indicates that the motions of both joints are in-phase, and any angle between these limits indicates an out-of-phase relation. DRP is a simple method that only analyzes one data point in the time series, which is not suitable for movements that exhibit coordination changes within a cycle. Another limitation of DRP is that it requires well-defined and consistent peaks, which may not be found in some dynamic movements or pathological systems.

Continuous relative phase (CRP) is a higher-order version of DRP that measures coordination based on phase planes. It involves spatio-temporal analysis of joint coupling by measuring the phase relationship between two interacting joints. A phase plane is constructed for each joint in which angular velocity $(\omega)$ is plotted against angular position $(\theta)$. Similar to the vector coding method, a phase angle (PA) is derived by the vector orientation relative to the right horizontal at each data point, expressed by Equation 2-2. The PA ranges from $0^{\circ}$ to $360^{\circ}$, but can be represented as $-180^{\circ}$ to $180^{\circ}$ or simply $0-180^{\circ}$. The CRP angle $\left(\mathrm{CRP}_{\theta}\right)$ is then calculated as the difference between the phase angles of both joints, given by Equation 2-3. The CRP method is better suited to analyze more complex coordination patterns because it consists of both spatial and temporal information, and provides a measure throughout the entire movement cycle.

$$
\begin{gathered}
P A=\tan ^{-1}\left(\frac{\omega_{i}}{\theta_{i}}\right) \\
C R P_{\theta}=P A_{\text {joint } 1}-P A_{\text {joint } 2}
\end{gathered}
$$

Hamill et al. [22] investigated lower extremity running injuries using CRP. The segment couplings that were analyzed were thigh flexion/extension and tibial rotation, tibial rotation and foot eversion/inversion, and femoral rotation and tibial rotation. A measure of between-trial variability for each subject was obtained by calculating the standard deviation of each point on the $\mathrm{CRP}_{\theta}$ ensemble curve, and then computing the average standard deviation over the complete time interval. Results showed that individuals with patellofemoral pain (PFP) had less variability in the CRP of the lower extremity segment couplings compared to uninjured individuals. The authors proposed that the pain present in the injured group caused these individuals to restrict the number of movement patterns utilized, which resulted in decreased variability. Miller et al. [71] also reported seeing less variability in the CRP of lower extremity segment couplings in injured runners with iliotibial band syndrome (ITBS). Interestingly, the same group of runners with ITBS had higher variability in the knee flexion/extension and foot adduction/abduction coupling compared to healthy runners. Similarly, Hein et al. [72] 
reported an increase in CRP variability for all examined couplings during the first half of stance in injured runners compared to healthy. However, no statistical significance was detected for this finding.

CRP has also been used to evaluate the phase relationships between joints or segments. In the same study by Hamill [22] that reported less variability in injured runners, there was a difference in thigh abduction/adduction and tibial rotation CRP pattern during mid-stance between the PFP and healthy groups that showed a more outof-phase coupling in the healthy group while the PFP group approached in-phase coordination. Conversely, Ferber et al. [46] reported an in-phase coordination pattern in healthy runners and an out-of-phase relationship in injured runners throughout the stance period. However, this was found for a different segment coupling (rear-foot eversion and tibial rotation) than that in the previous study. Lewek et al [73] analyzed stride to stride variability in knee motion in individuals with and without knee osteoarthritis (OA) using CRP. The uninvolved limb of the OA group displayed more variability in the frontal plane knee motion CRP compared to the involved and control limbs.

Despite its widespread use, there are several known limitations of CRP for measuring coordinative variability. The CRP method works under the assumption that data are sinusoidal [74], and it is well accepted that gait and other dynamic movements may deviate from this pattern. Even sinusoids having a frequency that is not $0.5 / \pi$ Hertz $(\mathrm{Hz})$ produce CRP results that are not equivalent to the relative temporal positions between the two signals as obtained in DRP [75]. Another limitation of CRP is the need to normalize the phase plots [76]. Normalization adjusts for differences in amplitude and frequency in the two signals $[70,76]$. Peters et al. [75] demonstrated the presence of artifacts in the form of a low frequency oscillation in the CRP of a non-normalized signal, and also showed differences in morphologies between two signals having different frequencies. The authors further reported that the CRP method did not produce the same phase relationship as in DRP for a constant time lag between two non-sinusoidal signals. Additionally, several different normalization techniques have been performed in a variety of studies using CRP. Van Emmerik and Wagenaar [77] normalized the angular position and velocity to their maximum and minimum values, but Burgess-Limerick [78] assigned the minimum and maximum values for angular position to -1 and 1 , respectively, and scaled the absolute maximum value of angular velocity to 1 while keeping zero velocity at the origin [70]. Other methods involving transforms and non-linear methods have also been used for normalization [75]. Discrepancies in normalization procedures make direct comparisons of results difficult. Contrarily, Kurz and Stergiou [79] showed that normalization changed the dynamic qualities of the phase portrait and are not convinced that normalizing is necessary. Lastly, CRP results are not easily interpretable to suggest a mechanical cause for pathology, and therefore, have limited clinical application [74].

\section{Vector Coding}

Hamill et al. [76] outlined the process of applying a vector coding technique adapted from Sparrow [67] as a means for measuring coordination based on relative 
motion. First, an angle-angle diagram is created such that the angular displacements of the two joints of interest are plotted along the $\mathrm{x}$ - and $\mathrm{y}$-axes. It is most common to plot the proximal joint along the $\mathrm{x}$-axis and the distal segment along the $\mathrm{y}$-axis. A coupling angle (CA) is obtained by calculating the angle between two adjacent data points on the angle-angle curve relative to the right horizontal, expressed by Equation 2-4. The CA ranges between $0^{\circ}$ and $360^{\circ}$. The $\mathrm{CA}$ is directional and thus requires the use of circular statistics to compute its mean value for a series of angles.

$$
C A=\tan ^{-1}\left(\frac{y_{i+1}-y_{i}}{x_{i+1}-x_{i}}\right)
$$

Heiderscheit et al. [23] chose to use a vector coding technique in their investigation of variability of joint coordination in individuals with unilateral PFP to avoid the limitations of CRP. The subject data from a previous study using CRP [22] was used to allow for comparison. The PFP and uninjured groups showed no difference in coordination variability when it was averaged across the full stride cycle, but the PFP group had less variability in the thigh rotation/leg rotation segment coupling at heel strike. Less variability seen in the PFP group agreed with the CRP interpretation. Chang et al. [80] further developed vector coding as a means to quantify and interpret joint coordination by categorizing ranges of coordination patterns, shown in Table 2-1. Needham et al. [81] utilized this classification scheme to analyze lumbar-pelvis coordination during gait by vector coding.

A few comparisons have been made between the use of vector coding and CRP. As previously mentioned, studies by Heiderscheit and Hamill $[22,23]$ using the same subject data but different methods for quantifying coordination variability did not reproduce exact results, but gave a similar interpretation that runners with PFP showed less variability in at least one segment coupling. Dierks and Davis [82] examined joint coupling relationships in uninjured runners using both vector coding and CRP techniques. Results obtained from vector coding showed CA values greater than $45^{\circ}$ for all periods of stance in three couplings (rearfoot eversion-inversion/tibial rotation, rearfoot eversioninversion/knee rotation, and tibial rotation/knee rotation), which indicated greater relative distal segment motion. However, CRP results revealed out-of-phase relationships for these couplings. Further, variability measured by vector coding was similar across all periods of stance for all couplings, while CRP variability was increased in the first and last periods. Miller et al. [71] performed a study to determine whether trends in coordination variability during movement are consistent between vector coding and CRP. CRP was found to produce a more conservative measure for variability than vector coding. One of the limitations of vector coding is the loss of higher-order information that may reduce its sensitivity. However, vector coding was said to have a more clinical application because interpretations can be made from the original positional signals. It was concluded that both CRP and vector coding may be valid methods for quantifying coordination and coordinative variability, but comparisons across these techniques may not be feasible. 
Table 2-1. Categories used to describe coordination patterns

\begin{tabular}{lr}
\hline \multicolumn{1}{c}{ Coordination Pattern } & Coupling Angle Range \\
\hline Anti-phase & $112.5^{\circ} \leq \mathrm{CA}<157.5^{\circ}, 292.5^{\circ} \leq \mathrm{CA}<337.5^{\circ}$ \\
In-Phase & $22.5^{\circ} \leq \mathrm{CA}<67.5^{\circ}, 202.5^{\circ} \leq \mathrm{CA}<247.5^{\circ}$ \\
Proximal & $0^{\circ} \leq \mathrm{CA}<22.5^{\circ}, 157.5^{\circ} \leq \mathrm{CA}<202.5^{\circ}, 337.5^{\circ} \leq \mathrm{CA} \leq 360^{\circ}$ \\
Distal & $67.5^{\circ} \leq \mathrm{CA}<112.5^{\circ}, 247.5^{\circ} \leq \mathrm{CA}<292.5^{\circ}$ \\
\hline
\end{tabular}

Reprinted with permission from Elsevier:

Chang, R. et al. Quantifying rearfoot-forefoot coordination in human walking. Journal of Biomechanics, 2008; 41(14): 3101-3105.

http://www.sciencedirect.com/science/article/pii/S0021929008003709 
The vector coding method was selected to quantify joint coordination in this thesis for several reasons. First, joint angular excursions in human gait are generally not sinusoidal oscillators. Vector coding has the capability to be applied to both sinusoidal and non-sinusoidal data, unlike CRP that may produce artificial oscillations in nonsinusoidal signals. Second, vector coding does not require normalization, which is a procedure that has been used in CRP analysis inconsistently and with some debate. Third, the $\mathrm{CA}$ may be more clinically relevant than the PA and $\mathrm{CRP}_{\theta}$ because physical rehabilitation is often based on the spatial relations between joints or segments. The CRP contains both displacement and velocity quantities, and its spatio-temporal information is not easily broken down to interpret contributions made exclusively by the spatial component. On the other hand, the CA is a spatial parameter that describes relative movement patterns between two joints or segments, and therefore, may be more useful in clinical a setting.

\section{Movement Coordination and Coordinative Variability in ACLR Individuals}

CRP was used to analyze relative phase dynamics in individuals with a reconstructed ACL during walking and running [83]. Sagittal plane motion in foot-shank and shank-thigh segment couplings was examined across the stance phase. The mean absolute value of the ensemble CRP curve values (MARP) was calculated as the average of the absolute values at all points on the ensemble curve. The ACLR group had a higher MARP than controls for the foot-shank coupling during walking, and a lower MARP for the shank-thigh coupling during walking and foot-shank coupling during running. While the timing of peaks on the foot-shank CRP curve was similar between both groups, the control group had a greater peak magnitude, which led the authors to suppose that coordination dynamics at the ankle joint were affected by ACL reconstruction. Also, the ACLR group demonstrated a more out-of-phase relationship in the shank-thigh coupling during late stance in walking.

Gribbin et al. [27] studied the effect of ACL reconstruction on the hip-knee joint coupling during walking and jogging using vector coding methods. Magnitude of excursion and CA were calculated across the gait cycle, and variability was defined as the consistency of magnitude and CA quantified on a scale from 0 to 1 , representing no variability and maximum variability, respectively. The magnitude of joint excursion for the hip transverse-knee frontal coupling was increased in the ACLR group during late swing, but decreased in all other couplings (hip frontal-knee frontal, hip frontal-knee sagittal, hip frontal-knee transverse, hip sagittal-knee frontal, and hip sagittal-knee transverse) during all sub-phases in walking compared to controls. CA values were increased in the ACLR group during all sub-phases of walking, and variability was also increased in the ACLR group during mid- and late stance phases of walking for all couplings except the hip sagittal-knee frontal and hip sagittal-knee transverse couples during mid-stance. Findings of decreased magnitude of joint excursion in the ACLR group suggested that the ACLR knee may experience constrained motion as a strategy for stabilizing the joint. Further, the authors proposed that the increased variability in the 
ACLR group could represent a struggle to find an optimal movement pattern in the ACLR limb.

In an effort to measure coordination variability in individuals with ACLR during a dynamic sport-specific task, Pollard et al. [84] evaluated lower extremity coupling in female soccer players with ACLR during a side-step cutting maneuver. A vector coding technique was used to calculate the CA for several couplings; hip rotation $/ \mathrm{knee}$ abduction-adduction, hip flexion-extension/knee abduction-adduction, hip rotation/ankle inversion-eversion, knee abduction-adduction/knee flexion-extension, knee abductionadduction/ankle inversion-eversion, knee abduction-adduction/knee rotation, and knee flexion-extension/knee rotation. Within-subject variability was derived by calculating the standard deviation of the CA at each percent of stance across trials for each subject, and only the first $40 \%$ of the stance period was analyzed to represent the event of early deceleration. The ACLR group showed increased variability compared to controls in the hip rotation/knee abduction-adduction, hip flexion-extension/knee abduction-adduction, knee abduction-adduction/knee flexion-extension, and knee abduction-adduction/knee rotation couplings. 


\section{CHAPTER 3. METHODOLOGY}

\section{Instrumented Gait Analysis}

The Motion Analysis Lab at the University of Tennessee Health Science Center (UTHSC) comprises an optoelectronic motion capture system (Qualisys, Gothenburg, Sweden) integrated with three force plates (AMTI, Watertown, MA, USA) embedded into the floor. Ten high-speed cameras track the motion of retroreflective markers placed on relevant bony landmarks, and the force plates measure ground reaction forces (GRF). The cameras emit infrared light that is reflected by the markers, and capture at a rate of $100 \mathrm{Hertz}(\mathrm{Hz})$. The force plates house a strain gauge in each corner configured in a Wheatstone bridge circuit that collects three-dimensional (3D) data. Force data is measured at a rate of $1000 \mathrm{~Hz}$. Software (Qualisys Track Manager, Qualisys, Gothenburg, Sweden) digitizes the 3D location of the markers in relation to a global coordinate system, which corresponds to the orientation of the capture volume or laboratory. Marker trajectories can then be exported to modeling and analysis software like Visual 3-D (V3D) (C-Motion, Inc., Germantown, MD) to derive joint angles and other model-based computations.

\section{Data Collection}

Institutional Review Board (IRB) approval was obtained prior to data collection, and all subjects signed an informed consent form. Twenty subjects (nine females, eleven males; body mass index (BMI) $25 \pm 3.5 \mathrm{~kg} / \mathrm{m}^{2}$ ) who had undergone unilateral ACLR (thirteen right, seven left) and been cleared to return to full activity were compared to twenty control subjects matched by gender and BMI (nine females, eleven males; BMI $22.4 \pm 2.4 \mathrm{~km} / \mathrm{m}^{2}$ ) (Table 3-1). The Centers for Disease Control and Prevention (CDC) divided the standard weight status categories into bins of $5 \mathrm{~kg} / \mathrm{m}^{2}$, so the mean BMI for the ACLR and control groups were considered to be within an acceptable range for comparison $(\mathrm{p}=0.0015)$ [85].

Kinetic and kinematic data were collected from all forty subjects during walking in the UTHSC Motion Analysis Laboratory. Retroreflective markers were attached to the subject for data collection. Calibration markers were placed on the following bony landmarks to define joint locations: the sacrum and bilaterally on the posterior superior iliac spine (PSIS), anterior superior iliac spine (ASIS), iliac crest, lateral femoral condyle, medial femoral condyle, lateral malleolus, medial malleolus, dorsum, fifth metatarsal, and first metatarsal. Tracking markers were attached in clusters of four on a rigid plate bilaterally to the lateral portions of the thigh and shank segments. A two-second static trial was captured for which the subject stood in a neutral position in the center of a force plate. Subsequently, motion trials were captured as the subject walked normally and at a self-selected speed (ACLR: $1.3 \pm 0.18 \mathrm{~m} / \mathrm{s}$, Control: $1.2 \pm 0.07 \mathrm{~m} / \mathrm{s}$ ) across the walking 
Table 3-1. Mean \pm SD for subject metrics for the ACLR and Control groups

\begin{tabular}{lcc}
\hline \multicolumn{1}{c}{ Metric } & ACLR & Control \\
\hline Gender & $9 \mathrm{~F} / 11 \mathrm{M}$ & $9 \mathrm{~F} / 11 \mathrm{M}$ \\
BMI $\left(\mathrm{kg} / \mathrm{m}^{2}\right)$ & $25 \pm 3.5$ & $22.4 \pm 2.4$ \\
Reconstructed/Matched Limb & $13 \mathrm{R} / 7 \mathrm{~L}$ & $13 \mathrm{R} / 7 \mathrm{~L}$ \\
\hline
\end{tabular}


platform. A gait trial was considered successful if the foot made full contact with at least one force plate. A minimum of three successful trials for each limb were collected for all subjects.

\section{D Modeling and Joint Angle Calculations}

V3D, a modeling and mathematical tool, was to analyze 3D motion data. Both static and motion files acquired from the motion capture system were imported into V3D to be assigned a biomechanical model. The following describes the detailed process of building the biomechanical model and the calculations used for deriving joint angles.

A standing calibration trial was used to generate a six DOF skeletal model consisting of seven rigid segments: pelvis, thighs, shanks, and feet. This model assumed the capability of each joint to exercise three translational and three rotational movements, for a total of six variables describing joint position and orientation. The inverse kinematics (IK) model was not used to avoid over-constraining the knee joint, which could hide the true behavior of the ACLR knee. A laboratory, or global coordinate system (GCS) was generated for which the Z-axis is vertical, the $\mathrm{Y}$-axis is positive in the direction of walking, and the $\mathrm{X}$-axis is perpendicular to the $\mathrm{Y}$ - and $\mathrm{Z}$-axes according to a right-handed Cartesian coordinate system. Each segment was assigned a fixed local coordinate system (LCS) that originates at its proximal end. Specific anatomical landmarks, which are based on marker location in the standing trial and described later, were used to establish the X-, Y-, and Z-axes that correspond to the individual LCS mediolateral, anterior-posterior, and inferior-superior axes, respectively. In this study, the pelvis was modeled as a CODA pelvis and its origin is the midpoint between the right and left anterior superior iliac spine (ASIS) markers. The transverse (XY) plane passes through both ASIS markers and the midpoint of the right and left posterior superior iliac spine (PSIS) markers. The X-axis of the pelvis LCS is oriented laterally and defined by the vector directed from the pelvis origin to the right ASIS. The Z-axis is oriented superiorly and defined perpendicular to the XY-plane. The Y-axis is defined as the cross product of the unit vectors representing the $\mathrm{X}$ - and $\mathrm{Z}$-axes, and is oriented anteriorly. The hip joint centers are automatically derived from the CODA pelvis and are estimates based on regression equations from Bell et al. [86]. The pelvis has cylindrical geometry while the remaining six segments are modeled as truncated cones. The LCS of the thigh, shank, and foot segments are established by first calculating segment endpoints. The Z-axis is the vector that extends superiorly from the distal endpoint to the proximal endpoint. The frontal (XZ) plane passes through the medial and lateral markers on the distal end and the joint center on the proximal end. The $\mathrm{Y}$-axis is directed anteriorly, and the $\mathrm{X}$-axis is the cross product of the $\mathrm{Y}$ - and Z-axes. For the thigh segment, the distal endpoint is the knee joint center, which is the midpoint between the medial and lateral femoral condyles. The thigh's proximal endpoint is the hip joint center. The Z-axis is the vector extending superiorly from the knee joint center to the hip joint center. The XZ plane passes through the medial and lateral femoral condyles on the distal end and the hip joint center on the proximal end. To obtain the anteriorly-directed Y-axis, the vector from the medial to lateral femoral condyle is crossed with the unit vector representing the Z-axis. The $\mathrm{X}$-axis 
is the cross product of the two unit vectors defining the $\mathrm{Y}$ - and Z-axes according to the right hand rule. For the shank, the distal endpoint is the ankle joint center, which is the midpoint between the medial and lateral malleoli. The proximal endpoint is the knee joint center. The Z-axis of the shank LCS is the vector extending from the ankle joint center to the knee joint center. The XZ plane passes through both malleoli markers and the knee joint center. The unit vector representing the $\mathrm{Z}$-axis is crossed with the unit vector directed from the medial to lateral malleolus to give the $\mathrm{Y}$-axis. The $\mathrm{X}$-axis is the cross product of the two unit vectors defining the Y-and Z-axes. For the foot LCS, the distal endpoint is defined by the fifth metatarsal and a standard radius of $0.6 \mathrm{~m}$. Its origin is the ankle joint center. The $Z$-axis is the vector extending from the distal endpoint to the ankle joint center. The $\mathrm{Y}$-axis is the cross product of the unit vector representing the $\mathrm{Z}$-axis and the vector from the distal joint center to the fifth metatarsal. The $\mathrm{X}$-axis is the cross product of the unit vectors defining the Y- and Z-axes. Unlike the thigh and shank LCS, the foot LCS is rotated such that the Z- and Y-axes are directed anterior and superior, respectively. Also, the distal and proximal joint centers are located at different heights, which cause an offset from $0^{\circ}$ of ankle dorsiflexion in what is considered the neutral position. A virtual foot was created to correct for this offset by adjusting the orientation of the foot LCS. In this method, the malleoli and fifth metatarsal are projected onto the floor so that they are in the same plane, and a modified foot LCS is created with these new virtual marker locations, as previously described. The modified foot LCS is oriented such that the Z-axis is directed superiorly and the $\mathrm{Y}$-axis is directed anteriorly.

A rotation matrix is constructed for each segment LCS that describes its orientation relative to the GCS and is used for joint angle calculations. A joint angle was defined as the orientation of the distal segment relative to the proximal segment. More specifically, a joint angle was obtained by transforming the distal segment LCS to the proximal segment LCS. Equation 3-1 [70] shows an example of deriving the rotation matrix of the knee joint by multiplying the shank (distal) LCS matrix by the transpose of the thigh (proximal) LCS matrix. Following the Cardan-Euler rotation sequence XYZ, the three angles extracted from the knee rotation matrix were flexion-extension (X), abduction-adduction (Y), and internal-external rotation (Z).

$$
\mathrm{R}_{\text {Knee }}=\mathrm{R}_{\text {Shank }} \mathrm{R}_{\text {Thigh }} \text {, }
$$

\section{Data Processing}

Qualisys Track Manager (QTM) software (Qualisys, Gothenburg, Sweden) was used to label the markers before exporting the data files to V3D. In V3D, a six DOF skeletal model consisting of seven rigid segments (pelvis, thighs, shanks, and feet) was applied to the kinematic data. The pelvis was modeled as a CODA pelvis, and hip joint centers were calculated according to Bell's equations [86]. The remaining six segments were modeled as frusta of right cones. Kinematic data were filtered using a low-pass fourth-order Butterworth filter with a cutoff frequency of $7 \mathrm{~Hz}$, and interpolated using a least-squares fit of a third-order polynomial over a maximum gap of ten data points. Force data were filtered using a low-pass fourth-order Butterworth filter with a cutoff 
frequency of $15 \mathrm{~Hz}$. Only the stance phase of the gait cycle was analyzed, which was defined as the period from heel strike to toe off. A $15 \mathrm{~N}$ threshold was assigned to the GRF curve to identify these two events. Joint angles for the hip, knee, and ankle for flexion-extension, abduction-adduction, and internal-external rotation were calculated according to the Cardan rotation sequence XYZ described previously.

Joint angle data derived within V3D was exported to Matlab (MathWorks, Massachusetts, USA). A custom script was created that calculated the CA according to the vector coding technique, described in Chapter 2. The CA was calculated for each joint coupling in both limbs of every subject for all three trials. Circular statistics were used to obtain the mean CA across each period for each subject trial, as well as the standard deviation across CA means which corresponded to the measure of within-subject variability. Group means for the reconstructed and contralateral limbs from the withinsubject variability values were obtained for the ACLR and control groups.

\section{Statistical Analyses}

Six joint couplings were selected for analysis based on kinematic differences found in the ACLR literature: hip abduction-adduction/knee abduction-adduction (HA/KA), hip abduction-adduction/knee rotation (HA/KR), hip flexion-extension/knee flexion-extension (HF/KF), hip rotation/knee abduction-adduction (HR/KA), hip rotation/knee rotation $(\mathrm{HR} / \mathrm{KR})$, and knee flexion-extension/ankle dorsiflexionplantarflexion (KF/ADF). The stance phase was further divided into four periods based on discrete events in the vertical GRF (vGRF) curve [82]. This was done to account for the changing functional demands (loading, weight acceptance, and propulsion) throughout this portion of the gait cycle. Initial loading (P1) was defined from heel strike (HS) to the first force peak to represent the loading due to impact force. Mid-stance (P2) was the interval between the first and second (maximum) force peaks, which corresponded to full bodyweight acceptance. Terminal stance (P3) was defined as the end of $\mathrm{P} 2$ to half the time to reach toe off (TO). The peak propulsive force typically occurs at the end of P3. Pre-swing (P4) began at the end of P3 and terminated at TO, which represented the unloading phase before swing occurs.

A Wilcoxon signed-rank test was used to compare the average variability of the reconstructed lower extremity between the ACLR and control groups for each joint coupling in each period. A second analysis was performed to consider both the reconstructed and contralateral limbs simultaneously. This was done by a multivariate analysis of variance (MANOVA) to compare the average variability between the ACLR and control groups for each coupling in each period. Cohen's $d$ was also calculated to evaluate the effect size of the observed differences. 


\section{CHAPTER 4. RESULTS}

\section{Coordination Variability}

The ACLR group exhibited greater joint coordination variability compared to the matched control group in several couplings during different periods of stance. Contrary to the original hypothesis that stated ACLR individuals would show decreased joint coordination variability in this study, results showed increased variability for the ACLR group in the following couplings: hip abduction-adduction/knee rotation and hip rotation/knee rotation in $\mathrm{P} 1$, and hip rotation/knee abduction-adduction and hip abduction-adduction/knee abduction- in P3. Significant findings for joint coordination variability are reported in Table 4-1. Post-hoc tests for select couplings were performed following the MANOVA, and results showed significant findings for the contralateral limb of the ACLR group compared to the reconstructed-match limb of the controls, reported in Table 4-2. Cohen's $d$ is included in both tables to provide a measure of effect size in addition to the p-value. A value equal to 0.20 suggests a relatively small effect, whereas values of 0.50 and 0.80 suggest a medium and large effect, respectively. A summary of all the measures of variability as well as coordination phase are reported in the Appendix (Tables A-1 and A-2). Figure 4-1 is an example of an angle-angle diagram for hip rotation/knee abduction-adduction from one pair of matched subjects and Figure 4-2 is the corresponding coupling angle plot. Figure 4-3 is a second example of an angle-angle diagram for the same subject pair but for hip rotation/knee rotation, and the corresponding coupling angle plot is in Figure 4-4. The ensemble average for CA variability is plotted in Figure 4-5.

\section{Coordination Phase}

The CA for each coupling in each period of stance was classified into one of four coordination phases based on the scheme developed by Chang et al. [80]. For the hipknee couplings, the hip had a greater contribution to motion relative to the knee in the proximal phase, and the opposite was true in the distal phase. The hip and knee rotated in the same direction if they were in-phase and in the opposite direction if they were antiphase. In the knee-ankle coupling, the knee was the proximal joint and the ankle was the distal joint. Differences in CA magnitude and classification between the ACLR and matched control reconstructed limbs are listed in Table 4-3. A proximal coordination pattern indicated more proximal joint (hip) contribution to motion relative to the distal joint (knee), whereas a distal coordination pattern indicated the inverse. An in-phase coordination pattern reflected motion in which both the proximal and distal joints rotated in either a positive or negative direction. For example, in-phase coordination for the hip abduction-adduction/knee rotation coupling during P2 was characterized by hip adduction and knee internal rotation. On the other hand, an anti-phase coordination pattern meant that the proximal and distal joints moved in opposition directions. For example, the hip rotation/knee abduction-adduction coupling displayed anti-phase 
Table 4-1. Joint coordination variability in the reconstructed and matched limb for ACLR versus Control

\begin{tabular}{lccccc}
\hline Coupling & Period & \multicolumn{2}{c}{$\begin{array}{c}\text { Variability (SD) } \\
\text { (Mean } \pm \text { Standard Deviation) }\end{array}$} & p-Value & Cohen's d \\
\cline { 3 - 4 } & & ACLR & Control & & \\
\hline HA/KR & 1 & $9.2 \pm 5.4$ & $6.3 \pm 3.0$ & 0.083 & 0.68 \\
$\mathrm{HA} / \mathrm{KA}$ & 1 & $7.3 \pm 7.0$ & $4.5 \pm 2.8$ & 0.123 & 0.52 \\
$\mathrm{HR} / \mathrm{KR}$ & 1 & $17.0 \pm 9.5$ & $10.8 \pm 6.9$ & 0.017 & 0.76 \\
$\mathrm{HA} / \mathrm{KR}$ & 2 & $28.4 \pm 17.9$ & $17.6 \pm 17.6$ & 0.048 & 0.61 \\
$\mathrm{HA} / \mathrm{KA}$ & 2 & $24.7 \pm 18.4$ & $15.1 \pm 12.1$ & 0.114 & 0.62 \\
$\mathrm{HA} / \mathrm{KA}$ & 3 & $8.0 \pm 6.3$ & $4.8 \pm 3.2$ & 0.097 & 0.64 \\
$\mathrm{HR} / \mathrm{KA}$ & 3 & $21.5 \pm 20.2$ & $9.0 \pm 6.0$ & 0.017 & 0.84 \\
$\mathrm{HR} / \mathrm{KR}$ & 3 & $22.5 \pm 17.9$ & $14.1 \pm 12.0$ & 0.114 & 0.56 \\
\hline
\end{tabular}

HA/KR: hip abduction-adduction/knee rotation; HAKA: hip abduction-adduction/knee abduction-adduction; HR/KR: hip rotation/knee rotation; HR/KA: hip rotation/knee abduction/adduction

Table 4-2. Joint coordination variability in the contralateral limb for ACLR versus the reconstructed-matched limb for Control

\begin{tabular}{lccccc}
\hline \multirow{2}{*}{ Coupling } & Period & \multicolumn{2}{c}{$\begin{array}{c}\text { Variability (SD) } \\
\text { (Mean } \pm \text { Standard Deviation) }\end{array}$} & p-Value & Cohen's d \\
\cline { 3 - 4 } & & \multicolumn{2}{c}{ ACLR } & Control & \\
\hline $\mathrm{HA} / \mathrm{KR}$ & 1 & $9.7 \pm 6.5$ & $6.3 \pm 3.0$ & 0.021 & 0.67 \\
$\mathrm{HR} / \mathrm{KR}$ & 1 & $15.5 \pm 9.3$ & $10.8 \pm 6.9$ & 0.044 & 0.58 \\
$\mathrm{HR} / \mathrm{KA}$ & 3 & $18.7 \pm 19.1$ & $9.0 \pm 6.0$ & 0.030 & 0.69 \\
\hline
\end{tabular}




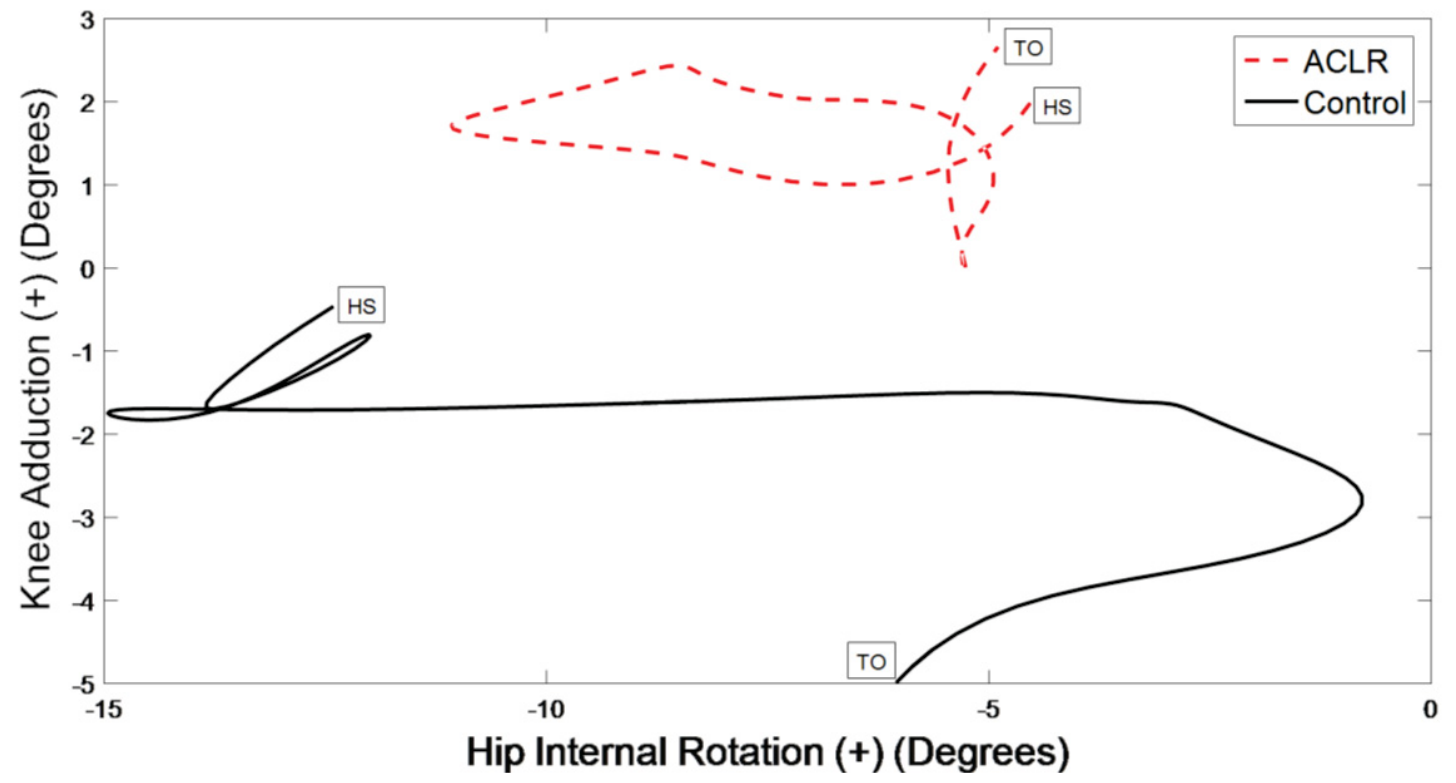

Figure 4-1. Angle-angle plot of the hip rotation/knee abduction-adduction coupling for one matched pair across the stance phase HS: heel strike; TO: toe off

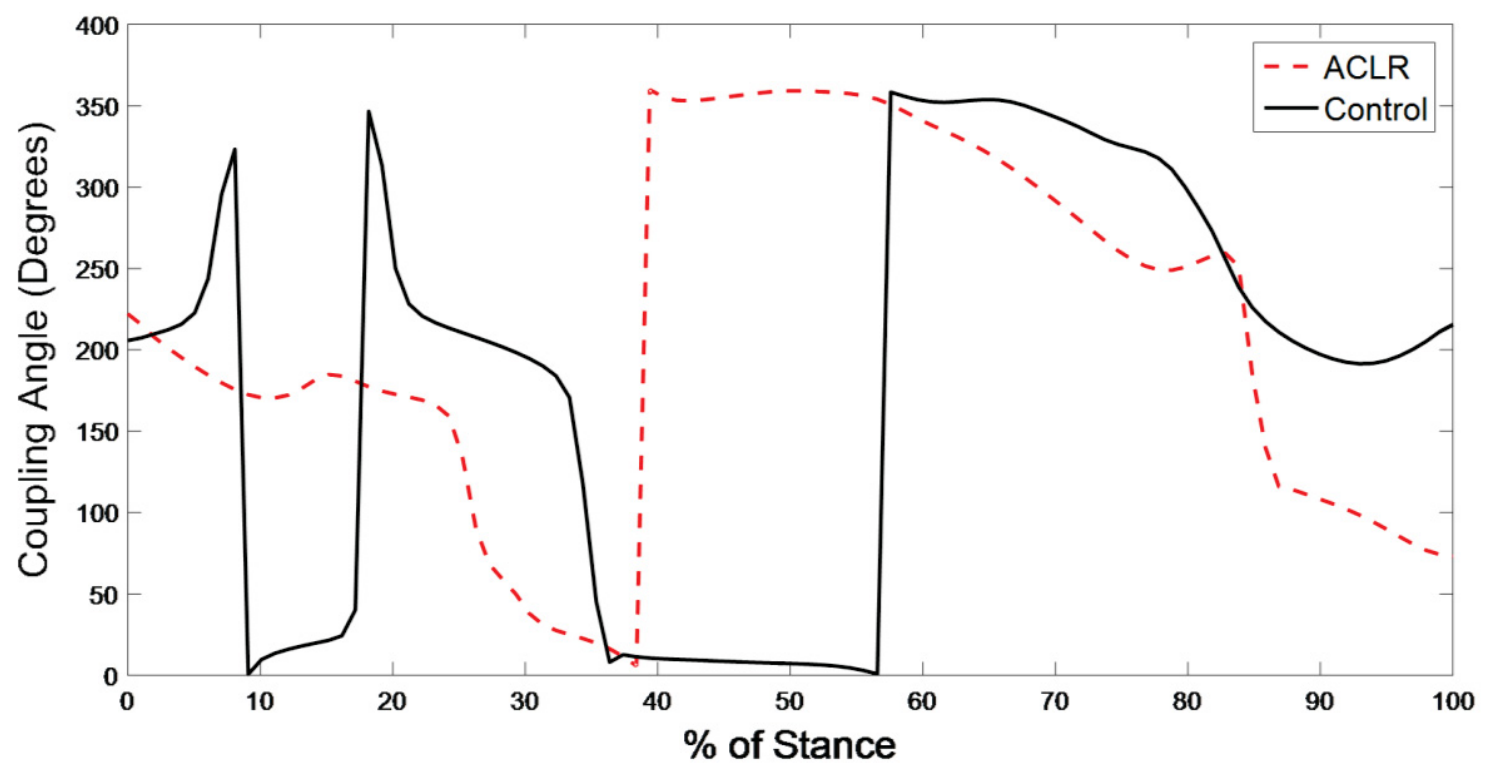

Figure 4-2. Coupling angle for hip rotation/knee abduction-adduction across the stance phase 


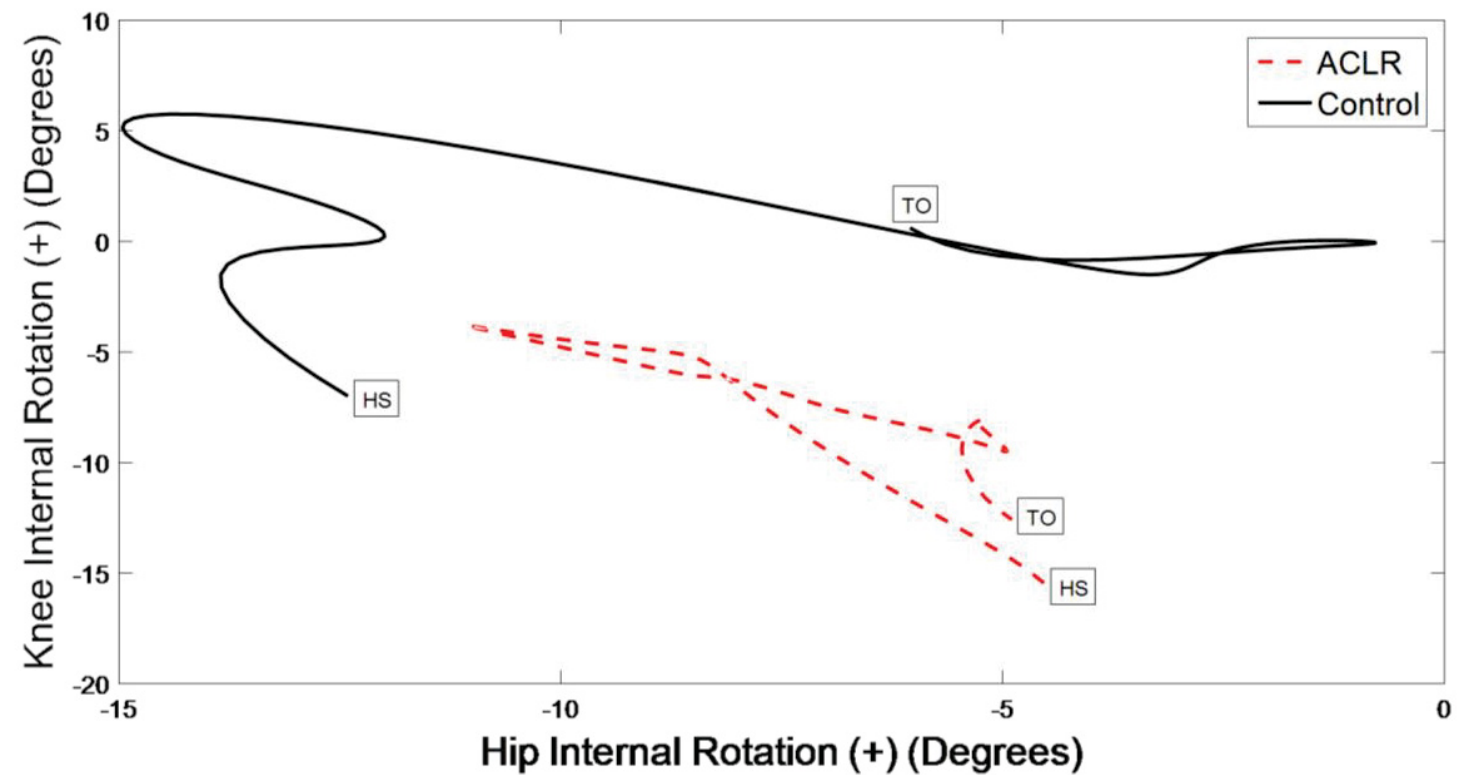

Figure 4-3. Angle-angle plot of the hip rotation/knee rotation coupling for one matched pair across the stance phase HS: heel strike; TO: toe off

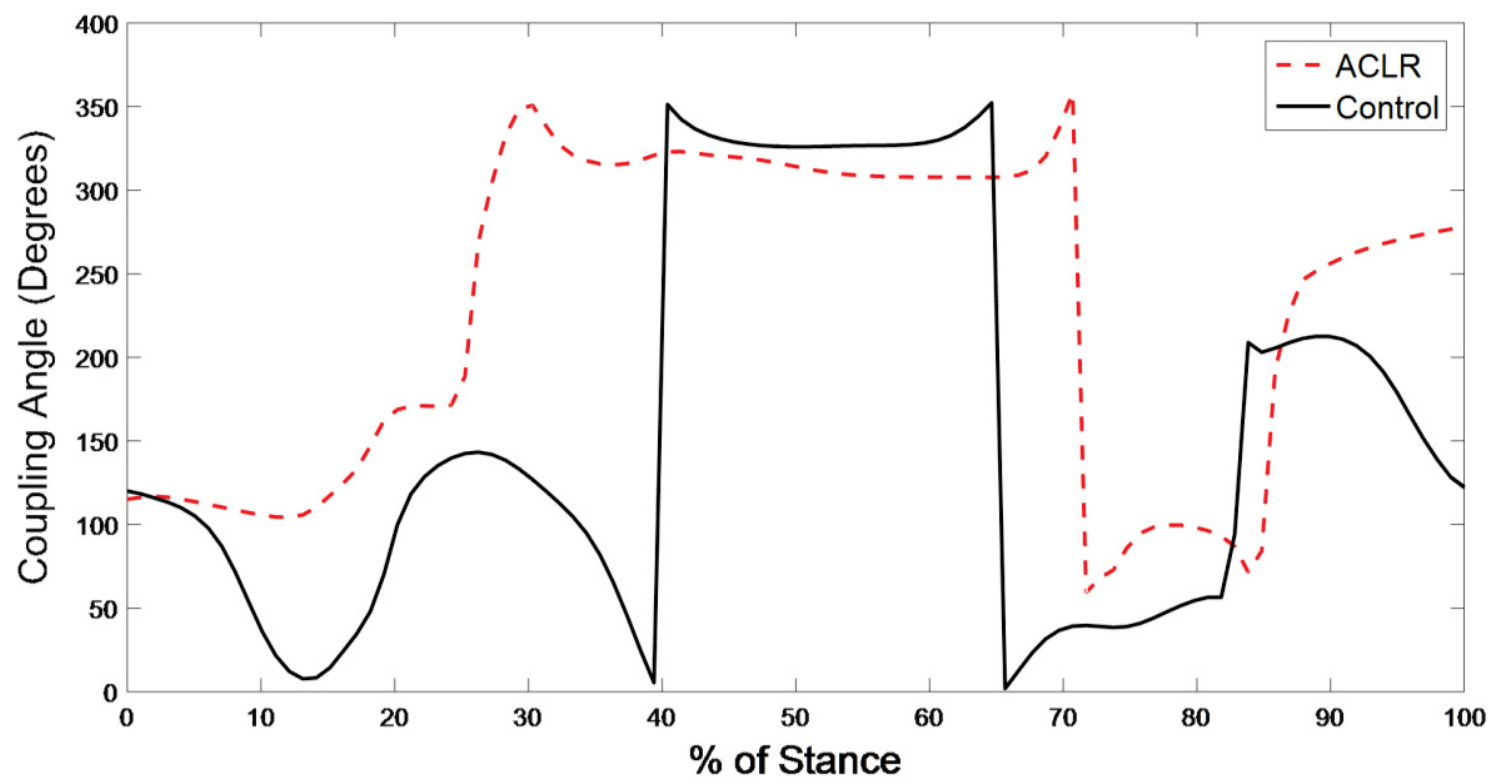

Figure 4-4. Coupling angle for hip rotation/knee rotation across the stance phase 


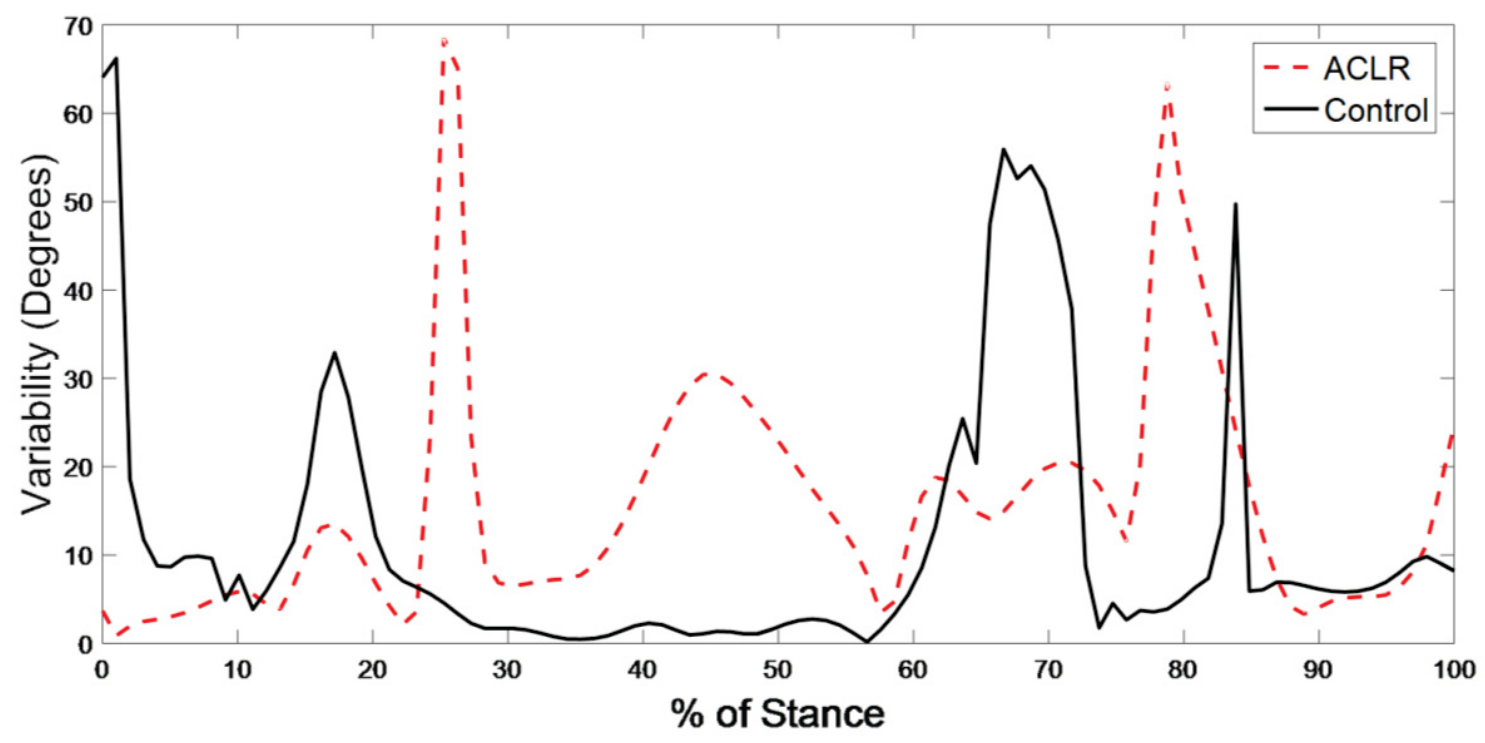

Figure 4-5. Ensemble average showing the coupling angle variability in hip rotation/knee abduction-adduction across the stance phase

Table 4-3. Joint coordination phase magnitude $\left(^{\circ}\right)$ and classification for the reconstructed and matched limbs in ACLR versus Control

\begin{tabular}{lccc}
\hline \multicolumn{1}{c}{ Coupling } & Period & \multicolumn{2}{c}{$\begin{array}{c}\left.\text { Coordination Phase } \mathbf{(}^{\circ}\right) \\
\text { (Mean/Classification) }\end{array}$} \\
\cline { 3 - 4 } & & ACLR & Control \\
\hline KF/ADF & 1 & 17/Proximal & 30/In-phase \\
HA/KA & 2 & 239/In-phase & $190 /$ Proximal \\
HA/KR & 2 & $230 /$ In-phase & 202/Proximal \\
HR/KA & 2 & 334/Anti-phase & $350 /$ Proximal \\
KF/ADF & 2 & $112 /$ Distal & 122/Anti-phase \\
HR/KA & 3 & 194/Proximal & 205/In-phase \\
HR/KA & 4 & 146/Anti-phase & $176 /$ Proximal \\
\hline
\end{tabular}

KF/ADF: knee flexion-extension/ankle dorsiflexion-plantarflexion 
coordination during P2 in which the hip showed internal rotation while the knee showed slight abduction. 


\section{CHAPTER 5. DISCUSSION}

The purpose of this study was to quantify joint coordination variability in individuals who have unilateral ACLR and compare their performance to gender- and BMI-matched control subjects during walking. Coordination phase was also assessed and classified into four categories to provide information about relative joint motion throughout stance. Vector coding was selected as the method for analysis because of the non-sinusoidal nature of gait, needless normalization procedure, straightforward application, and ability to translate the output to a clinically meaningful interpretation.

\section{Coordination Variability}

Contrary to the original hypothesis, the ACLR group demonstrated increased variability in the reconstructed limb for several joint couplings compared to the control group. Differences in coordination variability were found in the following joint couplings: hip rotation/knee rotation in P1, hip adduction-abduction/knee rotation in P2, and hip rotation/knee adduction-abduction in $\mathrm{P} 3$. These results suggest that despite meeting the criteria for return to sport, indicating a restoration of knee function and stability, individuals with ACLR exhibit altered coordinative function in their reconstructed lower extremity compared to non-injured controls. Coordination is a product of the dynamic interactions between the nervous system, musculoskeletal system, and environment, and the self-organized relations among these constituents are what allow for flexibility in movement patterns [87]. Therefore, deviations from normal coordination variability observed in ACLR individuals in the present study may be evidence of sensorimotor deficits relating to neuromuscular control.

A number of studies have examined joint coordination variability in an injured or diseased population. Our results opposed findings of decreased variability in individuals with unilateral PFP [23], Parkinson's disease [26], and low back pain [25]. However, the conflicting results may be attributed to the type of pathology present in the studied groups. PFP is an overuse injury, Parkinson's disease is a neurological disorder, and low back pain is commonly caused by muscle spasm, strain or intervertebral disc degeneration, and all three are chronic injuries and present with pain. On the other hand, an ACL tear results from excessive valgus loading of the knee and is an acute injury, and should not cause pain once repaired. Kiefer et al. [88] were able to discriminate between athletes with and without ACLR based on inter-segmental postural coordination. The athletes balanced on their reconstructed (or matched) leg and tracked anterior-posterior movement of a target that oscillated at low or high frequency on a computer screen. Phase angles for the hip and ankle were calculated according to a CRP method, and CRP variability was measured from the within-trial standard deviation. The ACLR group had more variability for the low-frequency target oscillation, which was expected because the slower movement induced by this condition is more challenging. The authors interpreted the higher variability in the ACLR group as instability in these athletes, and suggested a

decline in proprioceptive function post-reconstruction. Further discussion highlighted that 
graft tissue used to reconstruct the ligament does not consist of mechanoreceptors, which are responsible for sensing changes in joint load and position. The lack of sensory feedback in an ACLR system would thus hamper the ability to coordinate motion. Pollard et al. [84] also reported increased lower extremity variability in an ACLR subject group compared to controls, but during a side-step cutting maneuver. A vector coding method was used to obtain measures of coordination variability in select joint couplings of which hip rotation/knee abduction-adduction, hip flexion-extension/knee abduction-adduction, knee abduction-adduction/knee flexion-extension, and knee abduction-adduction/knee rotation showed differences between groups. In agreement with Kiefer et al. the authors offered altered neuromuscular control as a cause for the increased variability observed in the ACLR group. They also considered increased variability as a potential risk factor for re-injury or development of osteoarthritis. Individuals with ACLR in the present study similarly exhibited increased variability in the hip rotation/knee abduction-adduction coupling, but no other comparisons could be made because the remaining couplings were not analyzed.

Gribbin et al. [27] conducted a fairly congruent study to the one presented in this thesis. On the basis of dynamical systems theory, an injury may introduce new constraints to a movement system that affect the number of available DOF to complete a task. Accordingly, some movement patterns may no longer be accessible due to the newly imposed constraints, which might force an individual with ACLR to change their movement strategy. Therefore, hip-knee joint couplings were compared between ACLR and healthy individuals during walking to detect whether differences in variability exist. A vector coding method was used, and vector coding variability was defined as the consistency between the magnitude of joint excursion and coupling angle. Subjects walked on the treadmill at a set speed $(4.83 \mathrm{~km} / \mathrm{h})$ while kinetic and kinematic data were collected. Ten strides were analyzed for each subject, and the gait cycle was divided into loading response, mid-stance, late stance, and swing phases by percentages. The ACLR group demonstrated increased variability during mid- and late stance for hip abductionadduction/knee abduction-adduction, hip abduction-adduction/knee flexion-extension, hip abduction-adduction/knee rotation, and hip rotation/knee abduction-adduction couplings. Similarly, our study found increased variability in hip abductionadduction/knee rotation during mid-stance and hip rotation/knee abduction-adduction during terminal stance. Increased variability in hip rotation/knee rotation during the loading response was also detected in the ACLR group. However, a few discrepancies should be noted between both studies. Subjects in the former study walked on a treadmill at a prescribed speed and subjects in the present study walked along a platform at a selfselected pace. Also, different definitions of variability as well as gait phases were applied. Nonetheless, the overall interpretation of both sets of results was that individuals with ACLR had more variability in hip-knee coordination during walking compared to healthy individuals. Inconsistent motion displayed in the ACLR group could, again, reflect a deficit in sensorimotor control. The authors suggested that individuals with ACLR could not find optimal movement strategies and therefore, had not adapted to the new constraints triggered by ACL injury and reconstruction. 
Moraiti et al. [89] also reported an increase in gait variability in ACLR individuals compared to controls, but measured the largest Lyapunov Exponent (LE) to quantify variability instead of vector coding or CRP. This nonlinear analysis method considers variability to have a chaotic structure but also a deterministic pattern [60]. The maximal LE describes dynamic stability by first converting kinematic data from a time series to a phase space, then determining the rate of divergence of neighboring trajectories [62]. ACLR individuals had larger LE values for their reconstructed limbs than controls for knee flexion-extension during walking, which indicated a high amount of divergence and thus instability. The LE for a system that is stable is zero, which indicates minimal or no divergence in the trajectories, and is representative of a sinusoid $[89,90]$. The contralateral limb also had larger LE values in the ACLR group compared to controls, and this was considered a compensatory mechanism in the reconstructed population. The present study also found an increase in variability in the ACLR contralateral limb compared to the reconstructed-matched limb of the controls. This was for the hip rotation/knee abduction-adduction coupling during P3. Van Emmerik et al. [62] consider the LE method to work well for gait analysis because it does not assume periodic motion. Also, the LE describes the progression of locomotor stability over several consecutive strides, whereas each gait cycle is regarded as independent in other methods that measure variability relative to the mean. However, the LE is not a true measure of variability because it only quantifies system divergence while variation is also a quantity of convergence. Therefore, LE is better used to describe stability. Variability and stability have distinct definitions that relate to each other but are not synonymous [18]. Moreover, the LE method does not consider the interaction between joints like in vector coding, but rather the consistency of one joint motion over a series of cycles. Nonetheless, Moraiti et al. [89] reported a difference in knee stability between ACLR and control groups and related it to neuromuscular function, similar to the aforementioned studies.

Electromyography (EMG) is another method that has been used to assess changes in gait following ACL reconstruction. Although muscle weakness is expected after surgery, persistent quadriceps weakness has been reported in the ACLR population even at the time of return to sport [91]. Keays et al. [92] reported a significant correlation between quadriceps strength and knee joint stability, and Thomas et al. [93] reiterated that muscle weakness could contribute to the potential for re-injury by affecting movement strategies. Coats-Thomas et al. [94] compared muscle activity of the lower extremity between ACLR and ACL-intact individuals during a jump-cut maneuver. Muscle peak times and ratios of quadriceps/hamstrings and gastrocnemius/hamstrings muscle activity were obtained. Muscle peaks occurred faster in the ACL-intact group, and the quadriceps/hamstrings activity ratio was almost three times greater in ACLR individuals during the loading phase. The observed difference in muscle peak timings was related to a sensory deficit in the ACLR group, which could be due to impaired proprioception and damaged mechanoreceptors. An increase in the quadriceps/hamstrings activity ratio was indicative of a quadriceps-dominant activation pattern. Increased contraction of the quadriceps keeps the knee in a more extended position, which increases the risk for ACL injury. Therefore, this finding was suggested as a potential cause of reinjury in the ACLR population. 


\section{Coordination Phase}

Determining the amount of relative motion each joint contributes to a movement could reveal any compensatory or other mechanisms present as a result of injury or dysfunction within a system. Therefore, CA magnitude for each coupling in each period was classified into four types of coordination phases that describe relative joint motion. The study by Gribbin et al. [27] mentioned previously reported increased CAs in the ACLR group during walking compared to a healthy group, which suggested that the individuals with ACLR contributed more knee motion relative to the hip to accomplish a stride. This result was attributed to the hip acting as a primary stabilizer in order to promote knee joint stability. Specifically, an increased CA was observed in the hip abduction-adduction/knee flexion-extension, hip abduction-adduction/knee rotation, and hip flexion-extension/knee rotation couplings. Unfortunately, it is difficult to directly compare these findings to the coordination phases reported in this thesis because a different methodology was used in each study. The former study manipulated the CA range to be between $0^{\circ}$ and $90^{\circ}$. A CA less than $45^{\circ}$ was considered to represent greater hip motion relative to knee motion, and the opposite was true for a CA greater than $45^{\circ}$. A CA equal to $45^{\circ}$ indicated equal contribution of each joint to the overall movement. On the other hand, the $\mathrm{CA}$ fell between $0^{\circ}$ and $360^{\circ}$ for the present study. The larger CA range comprises more phase categories, which were defined in Chapter 2 but will be repeated here. A distal coordination pattern (CA from $67.5^{\circ}$ to $112.5^{\circ}$ and $246.5^{\circ}$ to $292.5^{\circ}$ ) described motion that is dominated by the distal joint of the coupling, which would be the knee in the case of a hip-knee relationship. A proximal coordination pattern (CA from $0^{\circ}$ to $22.5^{\circ}, 157.5^{\circ}$ to $202.5^{\circ}$, and $337.5^{\circ}$ to $360^{\circ}$ ) indicated the reverse, where the proximal joint is the primary contributor to motion. Additionally, anti-phase (CA from $112.5^{\circ}$ to $157.5^{\circ}$ and $292.5^{\circ}$ to $337.5^{\circ}$ ) and in-phase (CA from $22.5^{\circ}$ to $67.5^{\circ}$ and $202.5^{\circ}$ to $247.5^{\circ}$ ) coordination patterns specified that both joints rotated in the opposite or same direction, respectively.

The present study found several instances of altered coordination patterns between both groups. For the hip abduction-adduction/knee abduction-adduction coupling during mid-stance (P2), the ACLR group showed an in-phase relationship but controls demonstrated increased hip contribution. In normal gait the hip adducts in early stance and undergoes more frontal plane motion than the knee, which is consistent with the proximally dominant coordination pattern seen in the control group. However, the observed in-phase pattern in the ACLR group could support the idea offered by Gribbin et al. that the hip is acting as a stabilizer, and thus has constrained motion. The same coordination patterns were also found in the hip abduction-adduction/knee rotation coupling during P2, and a similar conclusion can be drawn. Since the knee is mostly extended during mid-stance, it does not experience much internal-external rotation. Therefore, the hip should contribute more frontal plane motion in this coupling. However, the ACLR group again exhibited decreased hip motion, indicative of a stabilizing role.

For the hip rotation/knee abduction-adduction coupling during P2, the control group maintained a proximally dominant coordination pattern but the ACLR group 
displayed anti-phase motion. The hip normally reaches peak internal rotation during midstance, and the knee exhibits minimal frontal plane motion throughout the gait cycle, which is reflected in the control coordination pattern. Conversely, the knee appeared to abduct slightly in the ACLR group while the hip was internally rotated, which is reflective of their anti-phase pattern. Interestingly, this coupling transitioned to a proximally dominant pattern in individuals with ACLR during terminal stance (P3) while controls exhibited in-phase motion. During terminal stance, the hip progresses towards maximal extension, and this is accompanied by internal rotation. Individuals with ACLR seemed to depend primarily on the hip during this stance period, avoiding frontal plane knee motion. On the contrary, the control group demonstrated both hip internal rotation and knee adduction. The more unstable position that occurs as the heel lifts off the ground during terminal stance possibly triggers individuals with ACLR to limit knee motion as a protective mechanism. Finally in pre-swing (P4), hip rotation/knee abduction-adduction was in anti-phase coordination in the ACLR group but controls demonstrated hip dominance. In this portion of stance, the hip begins to flex, and this is accompanied by external rotation. Results suggest that individuals with ACLR may be unable to control frontal motion at the knee prior to the swing phase. It is also possible that the hip has reduced rotational motion to help stabilize the knee.

Although no differences in variability between ACLR and control groups were observed in the knee-ankle coupling for flexion-extension, there was evidence of altered coordination patterns. In P1, knee flexion-extension/ankle dorsiflexion-plantarflexion was dominated proximally in individuals with ACLR but showed an in-phase relationship in controls. During the loading response (P1) in normal gait, the knee is extended at foot contact and progresses into slight flexion, and the ankle shifts from a neutral or dorsiflexed position into plantarflexion. More knee joint contribution relative to the ankle in ACLR individuals may indicate limited ankle range of motion (ROM), which has been associated with patellar tendinopathy $[51,52]$ and related to risk factors for ACL injury $[11,95,96]$. During mid-stance (P2), dominant joint contribution shifted distally in the ACLR group while the control group displayed anti-phase coordination. In typical gait, the knee transitions from flexion to extension during the mid-stance period and the ankle transitions from plantarflexion to dorsiflexion. The knee and ankle move in opposite directions, which is reflective of anti-phase motion and was observed in the control group. It is possible that ACLR knees are constrained during this period as a protective mechanism, or they may show stiffness as a result of reconstruction.

\section{Limitations}

It is important to acknowledge the limitations present in this study to allow for proper interpretations and comparisons of the results. Related to the ACLR subject demographic, ACL graft type and limb dominance were different within the group. Five subjects had bone-patellar tendon-bone autografts, one subject had a patellar tendon allograft, one subject had an Achilles allograft, three subjects had allografts but their origin was undocumented, and the graft choice for the remaining ten subjects was unknown. Webster et al. [97] showed that differences exist in external knee moments 
during walking depending on the type of graft used in ACL reconstruction (patellar tendon versus hamstring tendon), so is it possible that there could be a concomitant effect relating to graft type. However, Naendrup et al. [98] reported that all graft types used for ACL reconstruction produce similar kinematic outcomes in which anterior joint stability is restored. The present study also included subjects with ACL reconstructions on either the right or left side, without taking into account limb dominance. In a study examining lower limb dominance related to knee joint kinematics following ACL reconstruction [99], non-dominant ACLR knees demonstrated less extension and dominant ACLR knees demonstrated less varus rotation compared to their contralateral knees, respectively, during walking. However, Brown et al. [100] determined that lower extremity limb dominance did not have an effect on kinematic patterns during treadmill running in healthy subjects. Limb dominance should not have an effect on the interpretation of the present results because control limbs were matched by side and a statistical test (MANOVA) was performed that included all four limbs in one comparison.

Both female $(\mathrm{n}=18)$ and male $(\mathrm{n}=22)$ subjects participated in this study, but females are reportedly at an increased risk for incurring an ACL injury compared to males [101]. The gender gap in ACL incidence is a result of different anatomical, hormonal, and biomechanical factors [102] like bony alignment, menstrual cycle, and muscle strength, respectively. Barrett et al. [103] studied gender differences in the variability of lower extremity kinematics and showed that females had lower variability in several individual joint rotations at different gait speeds on the treadmill. Pollard et al. [48] evaluated gender differences in lower extremity coupling variability and found women had less variability in more than half of the joint couplings compared to men. Decker et al. [104] reported gender differences in kinematics and energy absorption strategies during landing, and Malinzak et al. [105] revealed differences in knee joint angles and thigh muscle activations in a variety of athletics tasks between female and male recreational athletes. However, the inclusion of gender-matched control subjects should account for any possible discrepancies between genders.

Soft tissue artifact (STA) is a prevalent source of error in human movement analysis, but a systematic review of the literature [106] reported that efforts to resolve this issue remain inconclusive. Benoit et al. [107] attempted to quantify the effect of STA on knee kinematics during gait using intra-cortical bone pins and skin markers. Results showed rotational errors up to $4.4^{\circ}$ between the two marker sets. Another study [108] claimed that sagittal plane rotations of the knee are similar between radiostereometric (invasive) and optical tracking (non-invasive) methods, while frontal and transverse plane rotations were less accurate for the optical system. Leardini et al. [109] confirmed that rotation in the sagittal plane, representing flexion-extension, can be determined reliably using an optoelectronic system. It was supposed that application of joint constraints to the 3D skeletal model may minimize STA error, however, prescribing constraints to a pathological system could hide inherent characteristics related to its abnormal function. The authors suggested assessing subject-specific patterns of STA in ad-hoc exercises or developing a general STA characterization based on a large series of measurements on different subject populations as possible methods for minimizing STA error. STA error is also considered to be larger for higher impact activities. Interestingly, Gao and Zheng 
[110] investigated translations and rotations of skin markers during level walking and argued that STA may have inter-subject similarity.

There are two types of models that are commonly used in biomechanics research. An inverse kinematics (IK) model sets joint constraints that restrict certain motion between segments. Reducing the DOF by adding joint constraints minimizes the effect of STA on the calculation of joint rotations [111], however, the prescribed motion produced by joint constraints may also conceal pathology. On the other hand, the 6DOF model allows all joint translations and rotations. The unconstrained approach may be more suitable for analyzing ACLR kinematics to avoid the risk of hiding abnormal motion of the reconstructed knee. Further, the knee has a floating axis about which abductionadduction occurs that is permitted in the 6DOF model, but would be restricted to a fixed axis in the IK model. Robinson et al. [112] compared the estimations of knee joint kinematics and kinetics derived from a 6DOF and IK model for a side-cutting maneuver. The 6DOF model included the trunk, pelvis, thighs, shanks, and feet, and each local coordinate system was obtained from a static trial. The IK model consisted of the same segments as in the 6DOF model, but the hip, knee, and ankle were assigned translational joint constraints and the trunk had 3 rotational DOF relative to the pelvis. Root mean square error (RMSE) was used to determine the similarity between both models in the calculation of knee angles and moments. Results showed that peak flexion and abduction moments were greater in the 6DOF model, and the largest RMSE was observed in the knee abduction-adduction angle; the 6DOF model showed that the knee was in abduction throughout the stance phase while the IK model showed that it was in adduction. However, the classification of ACL injury risk, which was based on peak frontal plane knee moments during weight acceptance, was consistent despite the reported kinematic and kinetic differences between both models. The observed differences in knee angles and moments were attributed to STA for the 6DOF model and joint constraints for the IK model. The present study used a $6 \mathrm{DOF}$ model to preserve true knee joint motion. The model showed no signs of disarticulating joints or severely aberrant motion, which are common indicators of STA. Moreover, motion trials consisted only of walking, which is a low-impact task, and more dynamic activities such as jumping and cutting are reportedly more likely to be influenced by STA [113]. Also, all subjects were within the normal range of BMI, so excessive STA was not expected.

Another modeling problem is defining the foot segment. In the present study the foot was modeled as a rigid, single segment. It has been reported that a single-segment foot model does not accurately represent the foot's complex bony anatomy or how it functions, specifically in inversion-eversion and abduction-adduction, and in the case of foot deformities, which were not present in this study. Despite this limitation, many current gait evaluations still employ a one-segment foot model [114]. Therefore, a singlesegment foot model was used, but only sagittal plane motion (dorsiflexion-plantarflexion) was analyzed.

Walking speed is often debated in gait analysis. A set walking speed may eliminate the possibility of having a concomitant effect, but subjects may also adjust their normal gait to achieve the prescribed speed. The present study had individuals walk at 
their preferred speed, which allowed them to walk naturally. Walking speeds for each group were compared using an independent two-sample t-test, and were not found to be significantly different $(\mathrm{p}=0.06)$.

Lastly, many studies that assessed joint coordination variability using vector coding or CRP divided the stance phase into intervals to get a better representation of variability within different stages of gait $[23,27,80]$. Intervals were commonly defined as a percentage of the stance phase. However, in the present study the stance phase was divided into four periods based on vGRF $[82,115]$. Consequently, periods were specific to each individual trial to accommodate differences in timing between subjects. This method was considered to provide a more sensitive representation of each sub-phase of gait.

\section{Conclusion}

This study comprised a total of forty-eight hypothesis tests. Multiple statistical comparisons increase the probability of making a Type I error, which results in a false positive claim. In other words, the null hypothesis is rejected in favor of the alternate hypothesis when the observed difference is in fact a product of random chance, and does not represent true variation between two populations. The goal of this study was to quantify and compare lower extremity joint coordination between ACLR and healthy individuals, and current literature pertaining to this objective is limited with respect to the use of vector coding for evaluation of gait in an ACLR subject group. Therefore, the broad investigation of joint coordination variability presented in this thesis provided a framework for future studies that should focus on hip-knee couplings in both frontal and transverse planes from heel strike to terminal stance. The inclusion of Cohen's $d$ further suggested a medium to large effect of ACLR on select measures of variability, and provided additional support for the pursuit of a better understanding of joint coordination in the ACLR population, specifically for hip rotation/knee abduction-adduction and hip rotation/knee rotation couplings.

Although this study evaluated joint coordination variability during walking, which is a low-impact activity that is not associated with risk for ACL injury, the reported findings may indicate that individuals with ACLR do not display normal coordinative function even in daily activities. The increased variability seen in individuals with ACLR during walking relative to normal may be an indicator for the risk of injury during more dynamic tasks like jumping and pivoting. Also, since the present study was retrospective it remains unknown whether increased coordination variability is a cause for, or effect of ACL injury and subsequent repair. Overall, individuals with ACLR were shown to exhibit increased joint coordination variability in several hip-knee couplings compared to matched controls during walking. Individuals with ACLR also displayed altered coordination patterns. The results of the present study suggest that coordinative function may not be fully restored in individuals with ACLR following rehabilitation and/or return to sport, or abnormal coordinative function may be an implication for ACL injury.

Further investigation that is prospective, focuses primarily on hip-knee coupled motion in 
frontal and transverse planes, includes assessment of EMG in addition to kinematics, involves both walking and more dynamic activities, and is specific to one graft type may contribute relevant information for improving ACL injury prevention and rehabilitation, and may supplement research relating to the development of osteoarthritis. 


\section{LIST OF REFERENCES}

1. Joseph, A.M., et al., A multisport epidemiologic comparison of anterior cruciate ligament injuries in high school athletics. Journal of athletic training, 2013. 48(6): p. 810-817.

2. Gage, B.E., et al., Epidemiology of 6.6 million knee injuries presenting to United States emergency departments from 1999 through 2008. Acad Emerg Med, 2012. 19(4): p. 378-85.

3. Gordon, M.D.a.S., M.E., Anterior cruciate ligament injuries, in Orthopaedic Knowledge Update: Sports Medicine 3, J.G. Garrick, Editor. 2004, American Academy of Orthopaedic Surgeons: Rosemont, IL. p. 169.

4. Ardern, C.L., et al., Return to sport following anterior cruciate ligament reconstruction surgery: a systematic review and meta-analysis of the state of play. Br J Sports Med, 2011. 45(7): p. 596-606.

5. Gillquist, J. and K. Messner, Anterior cruciate ligament reconstruction and the long-term incidence of gonarthrosis. Sports Med, 1999. 27(3): p. 143-56.

6. Paterno, M.V., et al., Incidence of Second ACL Injuries 2 Years After Primary ACL Reconstruction and Return to Sport. Am J Sports Med, 2014. 42(7): p. 156773.

7. Blackburn, J.T., M.F. Norcross, and D.A. Padua, Influences of hamstring stiffness and strength on anterior knee joint stability. Clinical Biomechanics, 2011. 26(3): p. 278-283.

8. Myer, G.D., et al., Biomechanics laboratory-based prediction algorithm to identify female athletes with high knee loads that increase risk of ACL injury. Br J Sports Med, 2011. 45(4): p. 245-52.

9. Boden, B.P., et al., Video analysis of anterior cruciate ligament injury: abnormalities in hip and ankle kinematics. Am J Sports Med, 2009. 37(2): p. 2529.

10. Paterno, M.V., et al., Biomechanical measures during landing and postural stability predict second anterior cruciate ligament injury after anterior cruciate ligament reconstruction and return to sport. Am J Sports Med, 2010. 38(10): p. 1968-78.

11. Hewett, T.E., et al., Biomechanical measures of neuromuscular control and valgus loading of the knee predict anterior cruciate ligament injury risk in female athletes: a prospective study. Am J Sports Med, 2005. 33(4): p. 492-501.

12. Imwalle, L.E., et al., Relationship between hip and knee kinematics in athletic women during cutting maneuvers: a possible link to noncontact anterior cruciate ligament injury and prevention. J Strength Cond Res, 2009. 23(8): p. 2223-30.

13. Hewett, T.E. and G.D. Myer, The Mechanistic Connection Between the Trunk, Knee, and Anterior Cruciate Ligament Injury. Exercise and sport sciences reviews, 2011.39(4): p. 161-166.

14. Hewett, T.E., G.D. Myer, and K.R. Ford, Anterior cruciate ligament injuries in female athletes: Part 1, mechanisms and risk factors. Am J Sports Med, 2006. 34(2): p. 299-311.

15. Bernshtein, N.A., The co-ordination and regulation of movements. 1967. 
16. Scott Kelso, J.A. and B. Tuller, A Dynamical Basis for Action Systems, in Handbook of Cognitive Neuroscience, M.S. Gazzaniga, Editor. 1984, Springer US: Boston, MA. p. 321-356.

17. Todorov, E. and M.I. Jordan, Optimal feedback control as a theory of motor coordination. Nat Neurosci, 2002. 5(11): p. 1226-1235.

18. Emmerik, R.E.A.v. and E.E.H.v. Wegen, On Variability and Stability in Human Movement. Journal of Applied Biomechanics, 2000. 16(4): p. 394-406.

19. Bartlett, R., Movement Variability and its Implications for Sports Scientists and Practitioners: An Overview. International Journal of Sports Science \& Coaching, 2008. 3(1): p. 113-124.

20. Wilson, C., et al., Coordination variability and skill development in expert triple jumpers. Sports Biomech, 2008. 7(1): p. 2-9.

21. Hamill, J., C. Palmer, and R.E. Van Emmerik, Coordinative variability and overuse injury. Sports Med Arthrosc Rehabil Ther Technol, 2012. 4(1): p. 45.

22. Hamill, J., et al., A dynamical systems approach to lower extremity running injuries. Clin Biomech (Bristol, Avon), 1999. 14(5): p. 297-308.

23. Heiderscheit, B.C., J. Hamill, and R.E.A.v. Emmerik, Variability of Stride Characteristics and Joint Coordination among Individuals with Unilateral Patellofemoral Pain. Journal of Applied Biomechanics, 2002. 18(2): p. 110-121.

24. Heiderscheit, B.C., Movement Variability as a Clinical Measure for Locomotion. Journal of Applied Biomechanics, 2000. 16(4): p. 419-427.

25. Seay, J.F., R.E.A. Van Emmerik, and J. Hamill, Low back pain status affects pelvis-trunk coordination and variability during walking and running. Clinical Biomechanics, 2011. 26(6): p. 572-578.

26. Van Emmerik, R.E.A., et al., Identification of axial rigidity during locomotion in parkinson disease. Archives of Physical Medicine and Rehabilitation, 1999. 80(2): p. 186-191.

27. Gribbin, T.C., et al., Differences in hip-knee joint coupling during gait after anterior cruciate ligament reconstruction. Clin Biomech (Bristol, Avon), 2016. 32: p. 64-71.

28. Liu-Ambrose, T., The anterior cruciate ligament and functional stability of the knee joint. British Columbia Medical Journal, 2003. 45(10): p. 495-499.

29. Smith, H.C., et al., Risk factors for anterior cruciate ligament injury: a review of the literature - part 1: neuromuscular and anatomic risk. Sports Health, 2012. 4(1): p. 69-78.

30. Smith, H.C., et al., Risk factors for anterior cruciate ligament injury: a review of the literature-part 2: hormonal, genetic, cognitive function, previous injury, and extrinsic risk factors. Sports Health, 2012. 4(2): p. 155-61.

31. Loudon, J.K., W. Jenkins, and K.L. Loudon, The relationship between static posture and ACL injury in female athletes. Journal of Orthopaedic \& Sports Physical Therapy, 1996. 24(2): p. 91-97.

32. Griffin, L.Y., et al., Noncontact anterior cruciate ligament injuries: risk factors and prevention strategies. J Am Acad Orthop Surg, 2000. 8(3): p. 141-50.

33. Griffin, L.Y., et al., Understanding and preventing noncontact anterior cruciate ligament injuries: a review of the Hunt Valley II meeting, January 2005. Am J Sports Med, 2006. 34(9): p. 1512-32. 
34. Boden, B.P., et al., Mechanisms of anterior cruciate ligament injury. Orthopedics, 2000. 23(6): p. 573-8.

35. Tran, A.A., et al., The effect of foot landing position on biomechanical risk factors associated with anterior cruciate ligament injury. J Exp Orthop, 2016. 3(1): p. 13.

36. Ford, K.R., G.D. Myer, and T.E. Hewett, Valgus knee motion during landing in high school female and male basketball players. Med Sci Sports Exerc, 2003. 35(10): p. 1745-50.

37. Olsen, O.E., et al., Injury mechanisms for anterior cruciate ligament injuries in team handball: a systematic video analysis. Am J Sports Med, 2004. 32(4): p. 1002-12.

38. Ireland, M.L., Anterior Cruciate Ligament Injury in Female Athletes: Epidemiology. Journal of Athletic Training, 1999. 34(2): p. 150-154.

39. $\mathrm{Yu}, \mathrm{B}$. and W.E. Garrett, Mechanisms of non-contact ACL injuries. Br J Sports Med, 2007. 41 Suppl 1: p. i47-51.

40. Reiman, M.P., L.A. Bolgla, and D. Lorenz, Hip functions influence on knee dysfunction: a proximal link to a distal problem. J Sport Rehabil, 2009. 18(1): p. 33-46.

41. Ireland, M.L., et al., Hip strength in females with and without patellofemoral pain. J Orthop Sports Phys Ther, 2003. 33(11): p. 671-6.

42. Robinson, R.L. and R.J. Nee, Analysis of hip strength in females seeking physical therapy treatment for unilateral patellofemoral pain syndrome. J Orthop Sports Phys Ther, 2007. 37(5): p. 232-8.

43. Bolgla, L.A., et al., Hip strength and hip and knee kinematics during stair descent in females with and without patellofemoral pain syndrome. J Orthop Sports Phys Ther, 2008. 38(1): p. 12-8.

44. Souza, R.B. and C.M. Powers, Differences in hip kinematics, muscle strength, and muscle activation between subjects with and without patellofemoral pain. $\mathrm{J}$ Orthop Sports Phys Ther, 2009. 39(1): p. 12-9.

45. Noehren, B., I. Davis, and J. Hamill, ASB clinical biomechanics award winner 2006 prospective study of the biomechanical factors associated with iliotibial band syndrome. Clin Biomech (Bristol, Avon), 2007. 22(9): p. 951-6.

46. Ferber, R., I.M. Davis, and D.S. Williams, 3rd, Gender differences in lower extremity mechanics during running. Clin Biomech (Bristol, Avon), 2003. 18(4): p. 350-7.

47. Lephart, S.M., et al., Gender differences in strength and lower extremity kinematics during landing. Clin Orthop Relat Res, 2002(401): p. 162-9.

48. Pollard, C.D., et al., Gender differences in lower extremity coupling variability during an unanticipated cutting maneuver. J Appl Biomech, 2005. 21(2): p. 14352.

49. Lawrence, R.K., 3rd, et al., Influences of hip external rotation strength on knee mechanics during single-leg drop landings in females. Clin Biomech (Bristol, Avon), 2008. 23(6): p. 806-13.

50. Devita, P. and W.A. Skelly, Effect of landing stiffness on joint kinetics and energetics in the lower extremity. Med Sci Sports Exerc, 1992. 24(1): p. 108-15. 
51. Malliaras, P., J.L. Cook, and P. Kent, Reduced ankle dorsiflexion range may increase the risk of patellar tendon injury among volleyball players. Journal of Science and Medicine in Sport, 2006. 9(4): p. 304-309.

52. Backman, L.J. and P. Danielson, Low range of ankle dorsiflexion predisposes for patellar tendinopathy in junior elite basketball players: a 1-year prospective study. Am J Sports Med, 2011. 39(12): p. 2626-33.

53. Mauntel, T.C., et al., The effects of lower extremity muscle activation and passive range of motion on single leg squat performance. J Strength Cond Res, 2013. 27(7): p. 1813-23.

54. Bell, D.R., D.A. Padua, and M.A. Clark, Muscle strength and flexibility characteristics of people displaying excessive medial knee displacement. Arch Phys Med Rehabil, 2008. 89(7): p. 1323-8.

55. Noehren, B., et al., Long-term gait deviations in anterior cruciate ligamentreconstructed females. Med Sci Sports Exerc, 2013. 45(7): p. 1340-7.

56. Czamara, A., et al., Kinematics of Rotation in Joints of the Lower Limbs and Pelvis during Gait: Early Results\&\#x2014;SB ACLR Approach versus DB ACLR Approach. BioMed Research International, 2015. 2015: p. 13.

57. Di Stasi, S.L., et al., Gait patterns differ between ACL-reconstructed athletes who pass return-to-sport criteria and those who fail. Am J Sports Med, 2013. 41(6): p. 1310-8.

58. Turvey, M.T., Coordination. Am Psychol, 1990. 45(8): p. 938-53.

59. Davids, K., et al., Movement systems as dynamical systems: the functional role of variability and its implications for sports medicine. Sports Med, 2003. 33(4): p. 245-60.

60. Stergiou, N., R. Harbourne, and J. Cavanaugh, Optimal movement variability: a new theoretical perspective for neurologic physical therapy. J Neurol Phys Ther, 2006. 30(3): p. 120-9.

61. Glazier, P., et al., The interface between biomechanics and motor control: Dynamic systems theory and the functional role of movement variability, in Movement System Variability. 2006, Human Kinetics. p. 49-69.

62. van Emmerik, R.E.A., et al., Comparing dynamical systems concepts and techniques for biomechanical analysis. Journal of Sport and Health Science, 2016. 5(1): p. 3-13.

63. Arutyunyan, G., G. VS, and M. Mirskii, Investigation of aiming at a target. BIOPHYSICS-USSR, 1968. 13(3): p. 642-+.

64. Grieve, D., Gait patterns and the speed of walking. Biomed Eng, 1968. 3: p. 119122.

65. Hershler, C. and M. Milner, Angle--angle diagrams in the assessment of locomotion. Am J Phys Med, 1980. 59(3): p. 109-25.

66. Whiting, W.C. and R.F. Zernicke, Correlation of movement patterns via pattern recognition. J Mot Behav, 1982. 14(2): p. 135-42.

67. Sparrow, W.A., et al., Using relative motion plots to measure changes in intralimb and inter-limb coordination. J Mot Behav, 1987. 19(1): p. 115-29.

68. Davids, K., S. Bennett, and K.M. Newell, Movement System Variability. 2006: Human Kinetics. 
69. Tepavac, D. and E.C. Field-Fote, Vector coding: A technique for quantification of intersegmental coupling in multicyclic behaviors. Journal of Applied

Biomechanics, 2001. 17(3): p. 259-270.

70. Robertson, G., et al., Research Methods in Biomechanics, 2E. 2013: Human Kinetics.

71. Miller, R.H., et al., Continuous relative phase variability during an exhaustive run in runners with a history of iliotibial band syndrome. J Appl Biomech, 2008. 24(3): p. 262-70.

72. Hein, T., et al., Using the variability of continuous relative phase as a measure to discriminate between healthy and injured runners. Hum Mov Sci, 2012. 31(3): p. 683-94.

73. Lewek, M.D., et al., Stride-to-stride variability of knee motion in patients with knee osteoarthritis. Gait Posture, 2006. 23(4): p. 505-11.

74. DeLeo, A.T., et al., Lower extremity joint coupling during running: a current update. Clin Biomech (Bristol, Avon), 2004. 19(10): p. 983-91.

75. Peters, B.T., et al., Limitations in the use and interpretation of continuous relative phase. J Biomech, 2003. 36(2): p. 271-4.

76. Hamill, J., J.M. Haddad, and W.J. McDermott, Issues in quantifying variability from a dynamical systems perspective. Journal of Applied Biomechanics, 2000. 16(4): p. 407-418.

77. van Emmerik, R.E.A. and R.C. Wagenaar, Effects of walking velocity on relative phase dynamics in the trunk in human walking. Journal of Biomechanics, 1996. 29(9): p. 1175-1184.

78. Burgess-Limerick, R., B. Abernethy, and R.J. Neal, Relative phase quantifies interjoint coordination. Journal of biomechanics, 1993. 26(1): p. 91-94.

79. Kurz, M.J. and N. Stergiou, Effect of normalization and phase angle calculations on continuous relative phase. J Biomech, 2002. 35(3): p. 369-74.

80. Chang, R., R. Van Emmerik, and J. Hamill, Quantifying rearfoot-forefoot coordination in human walking. J Biomech, 2008. 41(14): p. 3101-5.

81. Needham, R., R. Naemi, and N. Chockalingam, Quantifying lumbar\&\#x2013;pelvis coordination during gait using a modified vector coding technique. Journal of Biomechanics. 47(5): p. 1020-1026.

82. Dierks, T.A. and I. Davis, Discrete and continuous joint coupling relationships in uninjured recreational runners. Clin Biomech (Bristol, Avon), 2007. 22(5): p. 581-91.

83. Kurz, M.J., et al., The effect of anterior cruciate ligament recontruction on lower extremity relative phase dynamics during walking and running. Knee Surgery, Sports Traumatology, Arthroscopy, 2005. 13(2): p. 107-115.

84. Pollard, C.D., et al., Altered lower extremity movement variability in female soccer players during side-step cutting after anterior cruciate ligament reconstruction. Am J Sports Med, 2015. 43(2): p. 460-5.

85. Adult BMI. 2015 [cited 2017 April 12]; Available from: https://www.cdc.gov/healthyweight/assessing/bmi/adult_bmi/index.html.

86. Bell, A.L., R.A. Brand, and D.R. Pedersen, Prediction of hip joint centre location from external landmarks. Human Movement Science, 1989. 8(1): p. 3-16. 
87. Taga, G., Y. Yamaguchi, and H. Shimizu, Self-organized control of bipedal locomotion by neural oscillators in unpredictable environment. Biol Cybern, 1991. 65(3): p. 147-59.

88. Kiefer, A.W., et al., Inter-segmental Postural Coordination Measures Differentiate Athletes with ACL Reconstruction from Uninjured Athletes. Gait \& posture, 2013. 37(2): p. 149-153.

89. Moraiti, C.O., et al., Anterior cruciate ligament reconstruction results in alterations in gait variability. Gait Posture, 2010. 32(2): p. 169-75.

90. Myers, S.A., et al., Gait variability is altered in patients with peripheral arterial disease. J Vasc Surg, 2009. 49(4): p. 924-931.e1.

91. Palmieri-Smith, R.M., A.C. Thomas, and E.M. Wojtys, Maximizing quadriceps strength after ACL reconstruction. Clin Sports Med, 2008. 27(3): p. 405-24, viiix.

92. Keays, S.L., et al., The relationship between knee strength and functional stability before and after anterior cruciate ligament reconstruction. J Orthop Res, 2003. 21(2): p. 231-7.

93. Thomas, A.C., et al., Muscle Atrophy Contributes to Quadriceps Weakness after ACL Reconstruction. Journal of science and medicine in sport / Sports Medicine Australia, 2016. 19(1): p. 7-11.

94. Coats-Thomas, M.S., et al., Effects of ACL reconstruction surgery on muscle activity of the lower limb during a jump-cut maneuver in males and females. Journal of orthopaedic research : official publication of the Orthopaedic Research Society, 2013. 31(12): p. 10.1002/jor.22470.

95. Fong, C.M., et al., Ankle-dorsiflexion range of motion and landing biomechanics. J Athl Train, 2011. 46(1): p. 5-10.

96. Wahlstedt, C. and E. Rasmussen-Barr, Anterior cruciate ligament injury and ankle dorsiflexion. Knee Surg Sports Traumatol Arthrosc, 2015. 23(11): p. 32027.

97. Webster, K.E., et al., Gait Patterns After Anterior Cruciate Ligament Reconstruction Are Related to Graft Type. The American Journal of Sports Medicine, 2005. 33(2): p. 247-254.

98. Naendrup, J.-H., et al., Kinematic outcomes following ACL reconstruction. Current Reviews in Musculoskeletal Medicine, 2016. 9(4): p. 348-360.

99. Wang, H., J.E. Fleischli, and N. Nigel Zheng, Effect of lower limb dominance on knee joint kinematics after anterior cruciate ligament reconstruction. Clinical Biomechanics, 2012. 27(2): p. 170-175.

100. Brown, A.M., R.A. Zifchock, and H.J. Hillstrom, The effects of limb dominance and fatigue on running biomechanics. Gait Posture, 2014. 39(3): p. 915-9.

101. Stevenson, J.H., et al., Assessing the effectiveness of neuromuscular training programs in reducing the incidence of anterior cruciate ligament injuries in female athletes: a systematic review. Am J Sports Med, 2015. 43(2): p. 482-90.

102. Beynnon, B.D. and S.J. Shultz, Anatomic Alignment, Menstrual Cycle Phase, and The Risk of Anterior Cruciate Ligament Injury. Journal of Athletic Training, 2008. 43(5): p. 541-542. 
103. Barrett, R., M.V. Noordegraaf, and S. Morrison, Gender Differences in the Variability of Lower Extremity Kinematics During Treadmill Locomotion. Journal of Motor Behavior, 2008. 40(1): p. 62-70.

104. Decker, M.J., et al., Gender differences in lower extremity kinematics, kinetics and energy absorption during landing. Clin Biomech (Bristol, Avon), 2003. 18(7): p. 662-9.

105. Malinzak, R.A., et al., A comparison of knee joint motion patterns between men and women in selected athletic tasks. Clin Biomech (Bristol, Avon), 2001. 16(5): p. 438-45.

106. Peters, A., et al., Quantification of soft tissue artifact in lower limb human motion analysis: a systematic review. Gait Posture, 2010. 31(1): p. 1-8.

107. Benoit, D.L., et al., Effect of skin movement artifact on knee kinematics during gait and cutting motions measured in vivo. Gait \& Posture, 2006. 24(2): p. 152164.

108. Tranberg, R., et al., Simultaneous measurements of knee motion using an optical tracking system and radiostereometric analysis (RSA). Acta Orthop, 2011. 82(2): p. 171-6.

109. Leardini, A., et al., Human movement analysis using stereophotogrammetry: Part 3. Soft tissue artifact assessment and compensation. Gait \& Posture, 2005. 21(2): p. 212-225.

110. Gao, B. and N.N. Zheng, Investigation of soft tissue movement during level walking: translations and rotations of skin markers. J Biomech, 2008. 41(15): p. 3189-95.

111. Lu, T.W. and J.J. O'Connor, Bone position estimation from skin marker coordinates using global optimisation with joint constraints. J Biomech, 1999. 32(2): p. 129-34.

112. Robinson, M.A., et al., Impact of knee modeling approach on indicators and classification of anterior cruciate ligament injury risk. Med Sci Sports Exerc, 2014. 46(7): p. 1269-76.

113. Miranda, D.L., et al., Kinematic differences between optical motion capture and biplanar videoradiography during a jump-cut maneuver. Journal of Biomechanics, 2013. 46(3): p. 567-573.

114. Pothrat, C., et al., One- and multi-segment foot models lead to opposite results on ankle joint kinematics during gait: Implications for clinical assessment. Clinical Biomechanics, 2015. 30(5): p. 493-499.

115. Ferber, R., I.M. Davis, and D.S. Williams, 3rd, Effect of foot orthotics on rearfoot and tibia joint coupling patterns and variability. J Biomech, 2005. 38(3): p. 47783.

116. Ligaments of the knee. Accessed March 16, 2017, from http://www.newhealthadvisor.com/ligaments-of-the-knee.html 


\section{APPENDIX. SUMMARY OF JOINT COORDINATION AND JOINT COORDINATION VARIABILITY MEASURES}

Table A-1. Summary of joint coordination variability measures for all joint couplings and stance periods

\begin{tabular}{|c|c|c|c|c|c|}
\hline \multirow[t]{2}{*}{ Period } & \multirow{2}{*}{$\begin{array}{c}\text { Joint } \\
\text { Coupling }\end{array}$} & \multicolumn{4}{|c|}{ Variability $\left(^{\circ}\right)($ Mean \pm Standard Deviation) } \\
\hline & & ACLR & Control & ACL-C & Control-C \\
\hline \multirow[t]{6}{*}{1} & $\mathrm{HA} / \mathrm{KA}$ & $7.3 \pm 7.0$ & $4.5 \pm 2.8$ & $5.8 \pm 3.4$ & $5.4 \pm 3.3$ \\
\hline & $\mathrm{HA} / \mathrm{KR}$ & $9.2 \pm 5.4$ & $6.3 \pm 3.0$ & $9.7 \pm 6.5$ & $6.8 \pm 5.3$ \\
\hline & $\mathrm{HF} / \mathrm{KF}$ & $5.4 \pm 3.4$ & $5.6 \pm 3.5$ & $4.6 \pm 2.5$ & $6.1 \pm 3.7$ \\
\hline & $\mathrm{HR} / \mathrm{KA}$ & $20.2 \pm 19.3$ & $20.6 \pm 20.0$ & $21.7 \pm 16.4$ & $23.0 \pm 18.7$ \\
\hline & $\mathrm{HR} / \mathrm{KR}$ & $17.0 \pm 9.5$ & $10.8 \pm 6.9$ & $15.5 \pm 9.3$ & $14.4 \pm 13.1$ \\
\hline & $\mathrm{KF} / \mathrm{ADF}$ & $7.8 \pm 4.5$ & $5.8 \pm 2.8$ & $6.8 \pm 3.7$ & $9.2 \pm 13.5$ \\
\hline \multirow[t]{6}{*}{2} & $\mathrm{HA} / \mathrm{KA}$ & $24.7 \pm 18.4$ & $15.1 \pm 12.1$ & $16.2 \pm 15.5$ & $19.8 \pm 20.6$ \\
\hline & $\mathrm{HA} / \mathrm{KR}$ & $28.4 \pm 17.9$ & $17.6 \pm 17.6$ & $18.9 \pm 16.0$ & $18.1 \pm 14.2$ \\
\hline & $\mathrm{HF} / \mathrm{KF}$ & $2.8 \pm 1.9$ & $2.6 \pm 1.3$ & $2.3 \pm 1.8$ & $3.0 \pm 2.0$ \\
\hline & $\mathrm{HR} / \mathrm{KA}$ & $13.2 \pm 11.3$ & $13.5 \pm 13.7$ & $15.0 \pm 12.2$ & $21.4 \pm 21.3$ \\
\hline & $\mathrm{HR} / \mathrm{KR}$ & $17.7 \pm 14.8$ & $18.1 \pm 16.4$ & $16.9 \pm 14.7$ & $12.9 \pm 11.2$ \\
\hline & $\mathrm{KF} / \mathrm{ADF}$ & $7.5 \pm 5.7$ & $9.4 \pm 6.0$ & $6.4 \pm 2.8$ & $10.3 \pm 11.7$ \\
\hline \multirow[t]{6}{*}{3} & $\mathrm{HA} / \mathrm{KA}$ & $8.0 \pm 6.3$ & $4.8 \pm 3.2$ & $5.2 \pm 2.5$ & $5.3 \pm 2.6$ \\
\hline & $\mathrm{HA} / \mathrm{KR}$ & $10.2 \pm 6.0$ & $8.2 \pm 5.1$ & $9.6 \pm 4.6$ & $8.6 \pm 5.5$ \\
\hline & $\mathrm{HF} / \mathrm{KF}$ & $2.5 \pm 1.2$ & $2.9 \pm 2.7$ & $3.6 \pm 3.0$ & $3.0 \pm 4.3$ \\
\hline & $\mathrm{HR} / \mathrm{KA}$ & $21.5 \pm 20.2$ & $9.0 \pm 6.0$ & $18.8 \pm 19.1$ & $11.1 \pm 8.2$ \\
\hline & $\mathrm{HR} / \mathrm{KR}$ & $22.5 \pm 17.9$ & $14.1 \pm 12.0$ & $20.0 \pm 19.3$ & $13.5 \pm 15.2$ \\
\hline & $\mathrm{KF} / \mathrm{ADF}$ & $2.7 \pm 1.3$ & $3.3 \pm 1.8$ & $3.3 \pm 1.8$ & $3.2 \pm 2.8$ \\
\hline \multirow[t]{6}{*}{4} & $\mathrm{HA} / \mathrm{KA}$ & $3.4 \pm 2.6$ & $3.8 \pm 2.3$ & $4.7 \pm 2.8$ & $4.2 \pm 2.4$ \\
\hline & $\mathrm{HA} / \mathrm{KR}$ & $5.8 \pm 4.0$ & $6.7 \pm 3.9$ & $6.0 \pm 3.8$ & $8.1 \pm 6.0$ \\
\hline & $\mathrm{HF} / \mathrm{KF}$ & $0.8 \pm 0.5$ & $1.2 \pm 0.8$ & $1.3 \pm 0.9$ & $1.3 \pm 1.4$ \\
\hline & $\mathrm{HR} / \mathrm{KA}$ & $7.8 \pm 5.4$ & $6.4 \pm 4.3$ & $11.0 \pm 11.3$ & $8.0 \pm 6.9$ \\
\hline & $\mathrm{HR} / \mathrm{KR}$ & $8.1 \pm 8.9$ & $10.9 \pm 10.8$ & $9.5 \pm 13.3$ & $10.4 \pm 9.2$ \\
\hline & $\mathrm{KF} / \mathrm{ADF}$ & $1.8 \pm 1.1$ & $1.9 \pm 1.1$ & $1.7 \pm 0.9$ & $1.7 \pm 0.9$ \\
\hline
\end{tabular}


Table A-2. Summary of joint coordination patterns for all joint couplings and stance periods

\begin{tabular}{|c|c|c|c|}
\hline \multirow[t]{2}{*}{ Period } & \multirow{2}{*}{$\begin{array}{c}\text { Joint } \\
\text { Coupling }\end{array}$} & \multicolumn{2}{|c|}{ Coordination Phase (Mean $\left({ }^{\circ}\right) /$ Classification) } \\
\hline & & ACLR & Control \\
\hline \multirow[t]{6}{*}{1} & $\mathrm{HA} / \mathrm{KA}$ & 9/Proximal & 6/Proximal \\
\hline & $\mathrm{HA} / \mathrm{KR}$ & 32/In-phase & 32/In-phase \\
\hline & $\mathrm{HF} / \mathrm{KF}$ & 126/Anti-phase & 129/Anti-phase \\
\hline & $\mathrm{HR} / \mathrm{KA}$ & 81/Distal & 94/Distal \\
\hline & $\mathrm{HR} / \mathrm{KR}$ & 74/Distal & 72/Distal \\
\hline & $\mathrm{KF} / \mathrm{ADF}$ & 17/Proximal & 30/In-phase \\
\hline \multirow[t]{6}{*}{2} & $\mathrm{HA} / \mathrm{KA}$ & 239/In-phase & 190/Proximal \\
\hline & $\mathrm{HA} / \mathrm{KR}$ & 230/In-phase & 202/Proximal \\
\hline & $\mathrm{HF} / \mathrm{KF}$ & 184/Proximal & 188/Proximal \\
\hline & $\mathrm{HR} / \mathrm{KA}$ & 334/Anti-phase & 350/Proximal \\
\hline & $\mathrm{HR} / \mathrm{KR}$ & 358/Proximal & 3/Proximal \\
\hline & $\mathrm{KF} / \mathrm{ADF}$ & 112/Distal & 122/Anti-phase \\
\hline \multirow[t]{6}{*}{3} & $\mathrm{HA} / \mathrm{KA}$ & 189/Proximal & 196/Proximal \\
\hline & $\mathrm{HA} / \mathrm{KR}$ & 191/Proximal & 171/Proximal \\
\hline & $\mathrm{HF} / \mathrm{KF}$ & 95/Distal & 97/Distal \\
\hline & $\mathrm{HR} / \mathrm{KA}$ & 194/Proximal & 205/In-phase \\
\hline & $\mathrm{HR} / \mathrm{KR}$ & 202/Proximal & 170/Proximal \\
\hline & $\mathrm{KF} / \mathrm{ADF}$ & 332/Anti-phase & 332/Anti-phase \\
\hline \multirow[t]{6}{*}{4} & $\mathrm{HA} / \mathrm{KA}$ & 161/Proximal & 180/Proximal \\
\hline & $\mathrm{HA} / \mathrm{KR}$ & 223/In-phase & 209/In-phase \\
\hline & $\mathrm{HF} / \mathrm{KF}$ & 73/Distal & 71/Distal \\
\hline & $\mathrm{HR} / \mathrm{KA}$ & 146/Anti-phase & 176/Proximal \\
\hline & $\mathrm{HR} / \mathrm{KR}$ & 238/In-phase & 218/In-phase \\
\hline & $\mathrm{KF} / \mathrm{ADF}$ & 318/Anti-phase & 319/Anti-phase \\
\hline
\end{tabular}




\section{VITA}

Kylie Davis was born and raised in Montreal, QC, Canada in 1994. She earned a Bachelor of Science in Biomedical Engineering from the University of Memphis in May 2015. During this time, she captained the University of Memphis women's soccer team and was named an All-American Scholar-Athlete. She also played for Canada's youth national soccer team and participated in three FIFA Women's World Cup tournaments. Kylie remained in Memphis after graduation to pursue a Master's Degree in the Joint Graduate Program in Biomedical Engineering at the University of Tennessee Health Science Center and University of Memphis, from which she graduated in May 2017. Her area of focus was in biomechanics, especially relating to sports injuries and rehabilitation, and she was mentored by Dr. Brooke Sanford. 